\title{
BROWNIAN MOTION AT A SLOW POINT
}

\author{
MARTIN T. BARLOW AND EDWIN A. PERKINS
}

\begin{abstract}
If $c>1$ there are points $T(\omega)$ such that the piece of a Brownian path $B, X(t)=B(T+t)-B(T)$, lies within the square root boundaries $\pm c \sqrt{t}$. We study probabilistic and sample path properties of $X$. In particular, we show that $X$ is an inhomogeneous Markov process satisfying a certain stochastic differential equation, and we analyze the local behaviour of its local time at zero.
\end{abstract}

1. Introduction. Call $T(\omega)$ a slow point for a stochastic process $X_{t}$ if

$$
\limsup _{\delta \rightarrow 0+}|X(T+\delta)-X(T)| \delta^{-1 / 2}<\infty \quad \text { a.s. }
$$

The existence of slow points for the Brownian path was first shown by Kahane (1974). These results were refined by Davis (1983) and Greenwood and Perkins (1983), who showed there exist times at which the lim sup in (1.1) equals $c$ if $c>1$ but not if $c<1$. In fact there exist such times if $c=1$, but the situation in this case is more delicate (see Davis and Perkins (1985)). In this paper we study probabilistic and sample path properties of $B_{T+\delta}-B_{T}$, where $T$ is a slow point of the Brownian motion, $B$. Such a process is of interest not only because it arises naturally within a Brownian path but also because it is the weak limit of a sequence of random walks conditioned to stay inside square root boundaries (see Greenwood and Perkins (1984)).

To describe the setting of this paper, we recall some results from Greenwood and Perkins $(1983,1984)$. Assume $c_{1}<c_{2}$ are real constants and let $A$ denote the differential operator $\frac{1}{2}\left(d^{2} / d x^{2}-x d / d x\right)$. The Sturm-Liouville equation

$$
A \psi=-\lambda \psi, \quad \psi\left(c_{i}\right)=0, \quad i=1,2,
$$

has a sequence of distinct negative eigenvalues, $\left\{-\lambda_{n}\left(c_{1}, c_{2}\right) \mid n=0,1,2, \ldots\right\}$, decreasing to $-\infty$, whose corresponding (simple) eigenfunctions, $\left\{\psi_{n}\left(c_{1}, c_{2}\right)\right\}$, form a complete orthonormal system in $L^{2}\left(\left[c_{1}, c_{2}\right], m\right)$, where $m(d x)=e^{-x^{2} / 2} d x$. We may, and shall, take $\psi_{0}\left(c_{1}, c_{2}\right)$ to be strictly positive on $\left(c_{1}, c_{2}\right)$. Let

$$
\theta\left(c_{1}, c_{2}\right)=\int_{c_{1}}^{c_{2}} \psi_{0}\left(c_{1}, c_{2}\right)(x) d m(x) .
$$

$\lambda_{0}\left(c_{1}, c_{2}\right)$ is strictly increasing in $c_{1}$ and decreasing in $c_{2}$, and $\lambda_{0}(-1,1)=1$. These and further properties of $\left\{\lambda_{n}\right\}$ may be found in Perkins (1983, p. 371). We will

Received by the editors May 19, 1985. Some of these results were presented at the 808th meeting of the AMS at Northwestern University on November 12, 1983.

1980 Mathematics Subject Classification. Primary 60J65, 60G17; Secondary 60H05, 60H10, $60 \mathrm{~J} 55$.

Key words and phrases. Brownian motion, slow point, stochastic differential equation, grossissement d'une filtration, local time, stochastic integral. 
for the most part be interested in the case $\left(c_{1}, c_{2}\right)=(-c, c)$, where $c>1$, and therefore $\lambda_{0}(c)=\lambda_{0}(-c, c)<1$. We will write $\psi_{n}(c)$ and $\theta(c)$ for $\psi_{n}(-c, c)$ and $\theta(-c, c)$ and often suppress dependence on $c$, which, unless otherwise indicated, is assumed to exceed 1. Now let $\left\{X_{i} \mid i \in \mathbf{N}\right\}$ be i.i.d. mean zero random variables such that $E\left(X_{1}^{2}\right)=1$ and $E\left(X_{1}^{2} \log ^{+}\left(X_{1}\right)\right)<\infty\left(\log ^{+} x=(\log x) \vee 0\right)$. Introduce the following notations:

$$
\begin{aligned}
& S_{n}=\sum_{i=1}^{n} X_{i}, \quad T_{0}=0, \quad T_{i+1}=\min \left\{n>T_{i}|| S_{n}-S_{T_{i}} \mid>c\left(n-T_{i}\right)^{1 / 2}\right\} \\
& b_{n}=\Gamma\left(1-\lambda_{0}\right) P\left(T_{1}>n\right)^{-1}, \quad \begin{array}{r}
S^{(n)}(t) \\
=S_{[n t]} n^{-1 / 2} \\
([n t] \text { is the integer part of } n t)
\end{array} \\
& L_{n}(t)=\sum_{i=0}^{\infty} I\left(T_{i} n^{-1} \leq t\right) b_{n}^{-1} .
\end{aligned}
$$

If $v>0, D\left([0, v], \mathbf{R}^{d}\right)$ and $D\left([0, \infty), \mathbf{R}^{d}\right)$ denote the spaces of $\mathbf{R}^{d}$-valued rightcontinuous functions with left limits on $[0, v]$ and $[0, \infty)$, respectively, with the $J_{1^{-}}$ topology of Skorokhod (see Billingsley $\left[\mathbf{3}\right.$, p. 111]). Let $C\left([\alpha, \beta], \mathbf{R}^{d}\right)$ and $C\left([0, \infty), \mathbf{R}^{d}\right)$ denote the spaces of $\mathbf{R}^{d}$-valued continuous functions on $[\alpha, \beta]$ and $[0, \infty)$, respectively, with the topology of uniform convergence on compacts. If $d=1$, we simply write $D[0, v], C[0, \infty)$, etc. If $X(\cdot, \omega)$ is a stochastic process on a complete probability space with sample paths in $D\left([0, \infty), \mathbf{R}^{d}\right.$ ) (respectively, $\left.C\left([0, \infty), \mathbf{R}^{d}\right)\right)$, let $\left\{\mathcal{F}_{t}^{X} \mid t \geq 0\right\}$ denote the smallest filtration, satisfying the "usual hypotheses", for which $X$ is an adapted process, and let $P_{X}$ denote the law of $X$ on $D\left([0, \infty), \mathbf{R}^{d}\right)$ (respectively, $C\left([0, \infty), \mathbf{R}^{d}\right)$ ). We will also use the notation $\left\{\mathcal{F}_{u}^{X} \mid u \in \mathbf{R}\right\}$ in the case when $X$ is indexed by the real line.

Theorem 13 of Greenwood and Perkins (1983) shows that $\left(S^{(n)}, L_{n}\right)$ converges weakly on $D\left([0, \infty), \mathbf{R}^{2}\right)$ to a continuous process $(B, L)$. Let $(\Omega, \mathcal{F}, P)$ denote the completion of $\left(C\left([0, \infty), \mathbf{R}^{2}\right)\right.$, Borel sets, $\left.P_{(B, L)}\right)$ and $\mathcal{F}_{t}=\mathcal{F}_{t}^{(B, L)}$. The result mentioned above also shows that

(1.2a) $B$ is an $\left\{\mathcal{F}_{t}\right\}$-Brownian motion $\left(B_{0}=0\right)$.

(1.2b) $L$ is nondecreasing, and its right continous inverse, $\tau$, is a stable subordinator of index $\lambda_{0}$, scaled so that $E\left(e^{-u \tau(1)}\right)=e^{-u^{\lambda_{0}}}$.

(1.2c) For a.a. $\omega$ and all $t \geq 0$,

$$
\begin{gathered}
|B(\tau(t-)+u)-B(\tau(t-))|<c \sqrt{u} \quad \text { for } u \in(0, \Delta \tau(t)), \\
|B(\tau(t))-B(\tau(t-))|=c \sqrt{\Delta \tau(t)} .
\end{gathered}
$$

(1.2d) If $\tau^{-}(t)=\sup \{\tau(u) \mid \tau(u) \leq t\}, \tau^{+}(t)=\inf \{\tau(u) \mid \tau(u)>t\}$ $A(t)=t-\tau^{-}(t)$, and $Y(t)=B(t)-B\left(\tau^{-}(t)\right)$, then $\mathcal{F}_{t}^{(A, Y)}=\mathcal{F}_{t}$ and $(A, Y)$ is a homogeneous strong Markov process whose transition probabilities are given in Greenwood and Perkins (1983, p. 244).

Therefore $\left\{\tau\left(t^{-}\right) \mid \tau\left(t^{-}\right)<\tau(t)\right\}$ are all slow points for $B$. However, these are not all the slow points of $B$ or even all the slow points for which the left side of (1.1) is at most $c$, since the latter is a dense set of Hausdorff dimension $1-\lambda_{0}(c)>0$ [Perkins (1983)]. The above results allow us to decompose the Brownian path into 
excursions corresponding to the flat spots of $L$ and during which the path stays inside square root boundaries. It is these excursions that we wish to study.

For $v>0$ let

$$
U_{v}=\inf \{t \mid \Delta \tau(t)>v\}, \quad S_{v}=\tau\left(U_{v^{-}}\right) \quad \text { and } \quad X^{(v)}(t)=B\left(S_{v}+t\right)-B\left(S_{v}\right) .
$$

Then [Greenwood and Perkins (1984, Theorem 9, Corollary 11(a))]

$$
P\left(S^{(n)} \varepsilon \cdot \mid T_{1}>n v\right) \stackrel{\mathrm{w}}{\rightarrow} P\left(X^{(v)} \varepsilon \cdot\right) \quad \text { on } D[0, v] \text { as } n \rightarrow \infty
$$

$\left(\stackrel{w}{\rightarrow}\right.$ denotes weak convergence). The intuitive idea of considering $X^{(v)}$ as a Brownian motion conditioned to stay inside $[-c \sqrt{t}, c \sqrt{t}]$ for $t \leq v$ is reinforced by

THEOREM 1.1. If $B^{(n)}(\cdot)=B([n \cdot] / n)$, then

$$
\begin{aligned}
& P\left(B^{(n)} \varepsilon \cdot|| B(i / n) \mid \leq c(i / n)^{1 / 2} \text { for } i=0, \ldots,[n v]\right) \\
& \quad \stackrel{\mathrm{w}}{\rightarrow} P\left(X^{(v)} \varepsilon \cdot\right) \quad \text { on } D[0, v] \text { as } n \rightarrow \infty .
\end{aligned}
$$

PROOF. Let $\left\{X_{i}\right\}$ be normal random variables in (1.3).

Although many properties of $X^{(v)}$ may be inferred from the above results, its law has never been described explicitly. In $\S 2$ we show $X^{(v)}$ is an inhomogeneous strong Markov process, find its transition probabilities and show that, as $v \rightarrow \infty, X^{(v)}$ converges weakly to a process, $X^{\infty}$, that is in many ways simpler to study. In particular, $X^{\infty}$ is the unique solution of the stochastic differential equation

$$
X_{t}^{\infty}=\beta_{t}+\int_{0}^{t}\left(\frac{\psi_{0}^{\prime}}{\psi_{0}}\right)\left(\frac{X^{\infty}(s)}{\sqrt{s}}\right) \frac{d s}{\sqrt{s}},
$$

where $\beta$ is a Brownian motion (Theorem 2.6(b)). To obtain the analogous equation for $X^{(v)}$, we apply a "grossissement d'une filtration" [see Jeulin (1980)] in §3, which shows, in particular, that $X^{(v)}$ is a semimartingale.

In $\S 4$ we study the behaviour of the local time of $B$ at a slow point. If $L_{t}^{x}(Y)$ denotes the local time of a semimartingale $Y$ in the sense of Meyer (1976), then it is well known that

$$
\begin{aligned}
& \limsup _{\delta \rightarrow 0+} L_{\delta}^{0}(B)\left(2 \delta \log \log \frac{1}{\delta}\right)^{-1 / 2}=1 \text { a.s. } \\
& \liminf _{\delta \rightarrow 0+} L_{\delta}^{0}(B) \delta^{-1 / 2}\left(\log \frac{1}{\delta}\right)^{\theta}=\left\{\begin{array}{ll}
\infty & \text { if } \theta>1 \\
0 & \text { if } \theta \leq 1
\end{array}\right. \text { a.s. }
\end{aligned}
$$

These results follow from Lévy's equivalence between $L_{\delta}^{0}$ and $\sup _{s \leq \delta} B_{s}$, Khintchine's law of the iterated logarithm and Lévy's escape rate for the one-sided maximum [Lévy $(1939$, p. 334)]. At a slow point $T$, however, it is feasible that the conditioning could cause the local time at $B(T)$ to increase more rapidly and thus effect (1.4) or (1.5).

Notation. $\alpha(c)=\left[2\left(\lambda_{0}(0, c)-\lambda_{0}(-c, c)\right)\right]^{-1}, c>0$.

THEOREM 1.2. (a) $\alpha$ is strictly increasing on $(0, \infty)$ and increases to 1 as $c \rightarrow \infty$. 
(b) For a.a. $\omega$ and all $t$ such that $\tau_{t-}(\omega)<\tau_{t}(\omega)$,

$$
\limsup _{\delta \rightarrow 0+}\left(L_{\tau_{t-}+\delta}^{B\left(\tau_{t-}\right)}-L_{\tau_{t-}}^{B\left(\tau_{t-}\right)}\right)\left(2 \delta \log \log \frac{1}{\delta}\right)^{-1 / 2}=1,
$$

$$
\liminf _{\delta \rightarrow 0+}\left(L_{\tau_{t-}+\delta}^{B\left(\tau_{t-}\right)}-L_{\tau_{t-}}^{B\left(\tau_{t-}\right)}\right) \delta^{-1 / 2}\left(\log \frac{1}{\delta}\right)^{\theta}= \begin{cases}\infty & \text { if } \theta>\alpha(c), \\ 0 & \text { if } \theta \leq \alpha(c) .\end{cases}
$$

This is proved in $\S 4$ by analyzing the local time of $X^{\infty}$. The fact that conditioning the sample paths to lie inside square root boundaries has affected the lim inf but not the lim sup is not so surprising. The small values of $L_{t}^{0}$ occur during the long excursions from zero, and the conditioning clearly affects the asymptotic occurrence of such excursions. On the other hand, the large values of $L_{t}^{0}$ occur due to a preponderance of zeroes, and the conditioning has little effect on the process when it is at zero.

Finally, in $\S 5$ we study the behaviour of stochastic integrals at a slow point. The general question as to whether or not sample path singularities of $B(\cdot, \omega)$ are also singularities for the stochastic integral $H \cdot B(\cdot, \omega)$ is considered in Barlow and Perkins (1985). In particular, that work considers the sets of rapid and slow points for a process $Z$ defined by

$$
R(Z)=\left\{t\left|\limsup _{\delta \rightarrow 0+}\right| Z_{t+\delta}-Z_{t} \mid\left(\delta \log \frac{1}{\delta}\right)^{-1 / 2}>0\right\}
$$

and

$$
S(Z)=\left\{t\left|\limsup _{\delta \rightarrow 0+}\right| Z_{t+\delta}-Z_{t} \mid \delta^{-1 / 2}<\infty\right\},
$$

respectively, and shows that

(1.6) If $H(t, \omega)$ is nonzero, predictable, locally bounded and continous in $t$, then $R(H \cdot B)=R(B)$ a.s.

(1.7) If $H(t, \omega)$ is nonzero, predictable, locally bounded and satisfies

$$
|H(s)-H(t)| \leq K\left(\log (1 /|t-s|)^{-1 / 2} \text { for some } K>0,\right.
$$

then $S(H \cdot B)=S(B)$ a.s.

It is natural to ask if these results are best possible. In particular, are there cases where $R(H \cdot B)$ and $R(B)$ coincide a.s. but $S(H \cdot B) \neq S(B)$ a.s. Such an example is given in (b) of

THEOREM 1.3. (a) If $H$ is locally bounded, $\left\{\mathcal{F}_{t}\right\}$-predictable and satisfies

$$
\limsup _{\delta \rightarrow 0+}|H(t+\delta)-H(t)|\left(\log \log \frac{1}{\delta}\right)^{1 / 2}<\infty \quad \text { for all } t \geq 0 \text { a.s. }
$$

then $\left\{\tau_{t-} \mid \tau_{t-}<\tau_{t}, t \geq 0\right\} \subset S(H \cdot B)$ a.s.

(b) If $\sigma:[0, \infty) \rightarrow[0,1]$ is strictly increasing near 0 , continuous, $\sigma(0)=0, \sigma\left(t^{3}\right)$ is concave and $\lim _{t \rightarrow 0+} \sigma(t)\left(\log \log \frac{1}{t}\right)^{1 / 2}=\infty$, then there is a bounded $\left\{\mathcal{F}_{t}\right\}$ predictable process, $H$, such that

$$
\begin{aligned}
& \left|H_{s}-H_{t}\right| \leq K_{T}(\omega) \sigma(|t-s|) \\
& \quad \text { for all } 0 \leq s, t \leq T \text { and some } K_{T}(\omega)<\infty \text { for all } T>0 \text { a.s. }
\end{aligned}
$$


and

$$
\left\{\tau_{t-} \mid \tau_{t-}<\tau_{t}, t \geq 0\right\} \cap S(H \cdot B)=\varnothing \quad \text { a.s. }
$$

The proof is given in $\S 5$. The continuity condition in (1.7) is stronger than that in (a) but so is the conclusion. We conjecture that there are bounded predictable integrands, $H$, satisfying the hypotheses of (a) but for which $S(B) \not \subset S(H \cdot B)$.

It will be convenient to let $\tilde{B}=\left(\tilde{\Omega}, \tilde{\mathcal{F}}, \tilde{\mathcal{F}}_{t}, \tilde{B}_{t}, \theta_{t}, P^{x}\right)$ denote the canonical representation of Brownian motion on $\tilde{\Omega}=C[0, \infty)$. Recall that

$$
\tilde{Z}(u)=B\left(e^{u}-1\right) e^{-u / 2}
$$

is then an Ornstein-Uhlenbeck process with generator $A$.

For a given process $Z$, indexed by $[0, \infty)$ or $\mathbf{R}$, let

$$
T_{Z}(0)=\inf \left\{t \geq 0 \mid Z_{t}=0\right\} .
$$

$I(A)$ will denote the indicator function of the set $A$, and the value of $K$, used to denote several unimportant constants, may change from line to line.

2. The law of Brownian motion at a slow point. We start with a preliminary result, most of which is proved in Greenwood and Perkins (1983).

Proposition 2.1. (a) For every $v>0, X^{(v)}(t v) v^{-1 / 2}$ and $X^{(1)}(t)$ have the same law on $C[0,1]$.

(b)

$$
P\left(X^{(1)}(1) \leq y\right)=\int_{-c}^{y} \psi_{0}(c)(y) m(d y) \theta(c)^{-1}(|y| \leq c) .
$$

(c) If $U_{n}=\min \left\{i \in \mathbf{N}|| B(i / n) \mid>c(i / n)^{1 / 2}\right\}$, then there is a slowly varying (at $\infty)$ function $\pi$ such that

$$
P\left(U_{n}>k\right)=P\left(U_{1}>k\right)=\pi(k) k^{-\lambda_{0}(c)} .
$$

Proof. (a) Fix $v>0$ and define a new Brownian motion, $\hat{B}$, by

$$
B_{t}=(n /[n v])^{1 / 2} \hat{B}(t[n v] / n) .
$$

Let $\hat{U}_{n}$ be as in (c) above but with $\hat{B}$ in place of $B$. If $\phi$ is bounded and continuous on $D[0,1]$, then by Theorem 1.1 ,

$$
\begin{aligned}
E\left(\phi\left(X^{(1)}(\cdot)\right)\right) & =\lim _{n \rightarrow \infty} E\left(\phi\left(B\left(\frac{[[n v] \cdot]}{[n v]}\right)\right) \mid U_{[n v]}>[n v]\right) \\
& =\lim _{n \rightarrow \infty} E\left(\phi\left(\left(\frac{n}{[n v]}\right)^{1 / 2} \hat{B}\left(\frac{[n v[n v] / n v \cdot]}{n}\right)\right) \mid \hat{U}_{n}>[n v]\right) \\
& =E\left(\phi\left(v^{-1 / 2} X^{(v)}(v \cdot)\right)\right) \quad \text { (Theorem 1.1). }
\end{aligned}
$$

For (b) and (c), see [10, Theorem 5].

It is now a fairly straightforward matter to use the above and Theorem 1.1 to describe the law of $X^{(v)}$. 
THEOREM 2.2. $\left\{X^{(v)}(t) \mid t \in[0, v]\right\}$ is an inhomogeneous Markov process starting at zero. If $\phi$ is bounded and measurable, the transition functions

$$
P_{s, t}^{(v)} \phi(x)=E\left(\phi\left(X_{t}^{(v)}\right) \mid X_{s}^{(v)}=x\right)
$$

are given by

$$
\begin{gathered}
P_{s, t}^{(v)} \phi(x)=E^{x}\left(\phi\left(\tilde{B}_{t-s}\right)|| \tilde{B}_{u} \mid \leq c(u+s)^{1 / 2} \forall 0 \leq u \leq v-s\right), \\
\text { if } 0<s<t \text { and }|x|<c \sqrt{s}, \\
\begin{aligned}
P_{0, t}^{(v)} \phi(x)=\int_{-c}^{c} \phi\left(t^{1 / 2} y\right) P^{t^{1 / 2} y}\left(\left|\tilde{B}_{u}\right| \leq c(u+t)^{1 / 2} \forall 0 \leq u \leq v-t\right) \\
\times \psi_{0}(c)(y) m(d y) \theta(c)^{-1}(v / t)^{\lambda_{0}(c)}, \quad \text { if } 0<t .
\end{aligned}
\end{gathered}
$$

ProOF. Fix a bounded, continuous function, $\phi$, on the line and $t \in(0, v]$. Let $t_{n}=[n t], v_{n}=[n v]$ and

$$
\rho_{n}(y)=P^{y}\left(|\tilde{B}(i / n)| \leq c\left(i / n+t_{n} / n\right)^{1 / 2} \text { for } i=0,1, \ldots, v_{n}-t_{n}\right) .
$$

Theorem 1.1 implies

$$
\begin{aligned}
E\left(\phi\left(X^{(v)}(t)\right)\right)= & \lim _{n \rightarrow \infty} E\left(\phi ( B ( \frac { t _ { n } } { n } ) ) I \left(\left|B\left(\frac{i}{n}\right)\right| \leq c\left(\frac{i}{n}\right)^{1 / 2}\right.\right. \\
& \left.\left.\times P\left(U_{n}>t_{n}\right) P\left(U_{n}>v_{n}\right)^{-1} \quad \text { for } i=t_{n}, \ldots, v_{n}\right) \mid U_{n}>t_{n}\right) \\
= & \lim _{n \rightarrow \infty} E\left(\phi\left(B\left(\frac{t_{n}}{n}\right)\right) \rho_{n}\left(B\left(\frac{t_{n}}{n}\right)\right) \mid U_{n}>t_{n}\right) \frac{\pi\left(t_{n}\right)}{\pi\left(v_{n}\right)}\left(\frac{t_{n}}{v_{n}}\right)^{-\lambda_{0}}
\end{aligned}
$$

by Proposition 2.1(c). Use the fact that

$$
P^{y}\left(\left|\tilde{B}_{u}\right| \leq c(u+t)^{1 / 2} \forall u \leq v-t \text { but }\left|\tilde{B}_{u}\right|=c(u+t)^{1 / 2} \text { for some } u \leq v-t\right)=0
$$

to show that if $y_{n} \rightarrow y$ then $\rho_{n}\left(y_{n}\right) \rightarrow \rho_{t}(y)$, where

$$
\rho_{t}(y)=P^{y}\left(\left|\tilde{B}_{u}\right| \leq c(u+t)^{1 / 2} \forall u \leq v-t\right) .
$$

Therefore Theorem 5.5 of Billingsley (1968), Theorem 1.1, Proposition 2.1 and the above imply that

$$
\begin{aligned}
E\left(\phi\left(X^{(v)}(t)\right)\right) & =E\left(\phi\left(X^{(t)}(t)\right) \rho_{t}\left(X^{(t)}(t)\right)\right)(v / t)^{\lambda_{0}} \\
& =\int_{-c}^{c} \phi\left(t^{1 / 2} y\right) \rho_{t}\left(t^{1 / 2} y\right) \psi_{0}(y) m(d y) \theta^{-1}\left(\frac{v}{t}\right)^{\lambda_{0}} .
\end{aligned}
$$

Now, in addition, fix $0<s_{1}<\cdots<s_{j}=s<t$ and a bounded continuous nonnegative function, $\rho$, on $\mathbf{R}^{j}$. Let $s_{i, n}=\left[n s_{i}\right], s_{n}=[n s]$ and

$\tilde{\rho}_{n}(y)=E^{y}\left(\phi\left(\tilde{B}\left(\left(t_{n}-s_{n}\right) / n\right)\right) I\left(|\tilde{B}(i / n)| \leq c\left(\left(i+s_{n}\right) / n\right)^{1 / 2}\right.\right.$

$$
\text { for } \left.\left.i=0,1, \ldots, v_{n}-s_{n}\right)\right) \text {. }
$$


Then

$$
\begin{aligned}
& E\left(\phi\left(X_{t}^{(v)}\right) \rho\left(X_{s_{1}}^{(v)}, \ldots, X_{s_{j}}^{(v)}\right)\right) \\
&=\lim _{n \rightarrow \infty} E\left(\rho\left(B\left(\frac{s_{1, n}}{n}\right), \ldots, B\left(\frac{s_{j, n}}{n}\right)\right) \tilde{\rho}_{n}\left(B\left(\frac{s_{n}}{n}\right)\right) \mid U_{n}>s_{n}\right) \\
& \times P\left(U_{n}>s_{n}\right) P\left(U_{n}>v_{n}\right)^{-1} \\
&= E\left(\rho\left(X_{s_{1}}^{(s)}, \ldots, X_{s_{j}}^{(s)}\right) \tilde{\rho}\left(X_{s}^{(s)}\right)\right)(v / s)^{\lambda_{0}}
\end{aligned}
$$

where

$$
\tilde{\rho}(y)=E^{y}\left(\phi(\tilde{B}(t-s)) I\left(|\tilde{B}(u)| \leq c(u+s)^{1 / 2} \forall u \leq v-s\right)\right),
$$

and we have used Theorem 1.1, Proposition 2.1 and [3, Theorem 5.5] as before. If $\phi \equiv 1$, we get

$$
E\left(\rho\left(X_{s_{1}}^{(v)}, \ldots, X_{s_{j}}^{(v)}\right)\right)=E\left(\rho\left(X_{s_{1}}^{(s)}, \ldots, X_{s_{j}}^{(s)}\right) \rho_{s}\left(X_{s}^{(s)}\right)\right)(v / s)^{\lambda_{0}} .
$$

By replacing $\rho$ with $\rho\left(y_{1}, \ldots, y_{j}\right) \tilde{\rho}\left(y_{j}\right) / \rho_{s}\left(y_{j}\right)$ in (2.5) (note that (2.5) now holds for any nonnegative measurable $\rho$ ), and combining this with (2.4) we obtain

$$
\begin{aligned}
& E\left(\phi\left(X_{t}^{(v)}\right) \rho\left(X_{s_{1}}^{(v)}, \ldots, X_{s_{j}}^{(v)}\right)\right)=E\left(\rho\left(X_{s_{1}}^{(v)}, \ldots, X_{s_{j}}^{(v)}\right) \tilde{\rho}\left(X_{s}^{(v)}\right) \rho_{s}\left(X_{s}^{(v)}\right)^{-1}\right) \\
& \Rightarrow E\left(\phi\left(X_{t}^{(v)}\right) \mid X_{u}^{(v)}, u \leq s\right)=\frac{\tilde{\rho}}{\rho_{s}}\left(X_{s}^{(v)}\right) \\
& =E^{X_{s}^{(v)}}\left(\phi\left(\tilde{B}_{t-s}\right)|| \tilde{B}_{u} \mid \leq c(u+s)^{1 / 2} \forall u \leq v-s\right) \quad \text { a.s. }
\end{aligned}
$$

This proves (2.1), and since (2.2) is immediate from (2.3), the proof is complete.

Notation. If $c_{1}<c_{2}$, let

$$
\rho\left(c_{1}, c_{2}\right)=\inf \left\{t \mid \tilde{Z}_{t} \notin\left[c_{1}, c_{2}\right]\right\}
$$

and write $\rho(c)$ (or $\rho$ ) for $\rho(-c, c)$ as usual.

It is well known (e.g. see Perkins (1983, Lemma 10(a)) and Proposition 2.4 below) that for some $K=K\left(c_{1}, c_{2}\right)$,

$$
\begin{aligned}
P^{x}\left(\rho\left(c_{1}, c_{2}\right)>t\right)=e^{-\lambda_{0}\left(c_{1}, c_{2}\right) t}\left(\theta\left(c_{1}, c_{2}\right) \psi_{0}\left(c_{1}, c_{2}\right)(x)+r(t, x)\right) \\
\text { where }|r(t, x)| \leq K e^{-\left(\lambda_{1}\left(c_{1}, c_{2}\right)-\lambda_{0}\left(c_{1}, c_{2}\right)\right) t}
\end{aligned}
$$

THEOREM 2.3. (a) If $0<s<v$, then $P_{X^{(s)}}$ and $\left.P_{X^{(v)}}\right|_{C[0, s]}$ are equivalent probabilities on $C[0, s]$ with

$$
\left.\frac{d P_{X^{(v)}}}{d P_{X^{(s)}}}\right|_{C[0, s]}=P^{X_{s}^{(s)} / \sqrt{s}}\left(\rho(c)>\log \left(\frac{v}{s}\right)\right)\left(\frac{v}{s}\right)^{\lambda_{0}(c)} .
$$

(b) $X^{(v)} \stackrel{\mathrm{w}}{\rightarrow} X^{\infty}$ on $C[0, \infty)$ as $v \rightarrow \infty$, where the law of $X^{\infty}$ is given by

$$
\left.\frac{d P_{X^{\infty}}}{d P_{X^{(s)}}}\right|_{C[0, s]}=\psi_{0}\left(\frac{X_{s}^{(s)}}{\sqrt{s}}\right) \theta>0 \quad P_{X}(s)-a . s .
$$

Moreover, $X^{\infty}$ is an inhomogeneous Markov process starting at zero with transition functions

$$
\begin{aligned}
P_{s, t} \phi(x)= & E^{x / \sqrt{s}}\left(\phi(\sqrt{t} \tilde{Z}(\log t / s)) \psi_{0}(\tilde{Z}(\log t / s)) I(\rho>\log t / s)\right) \\
& \times \psi_{0}(x / \sqrt{s})^{-1}(t / s)^{\lambda_{0}}, \quad \text { if } 0<s<t,|x|<c \sqrt{s},
\end{aligned}
$$




$$
P_{0, t} \phi(0)=\int_{-c}^{c} \phi(\sqrt{t} y) \psi_{0}(y)^{2} m(d y) .
$$

Finally,

$$
\left|X_{s}^{\infty}\right|<c \sqrt{s} \text { for all } s>0 \text { a.s. }
$$

PROOF. (a) This is immediate from (2.5) and a short computation.

(b) (2.6) and the above show that for each $s>0$,

$$
\left.\lim _{v \rightarrow \infty} \frac{d P_{X^{(v)}}}{d P_{X^{(s)}}}\right|_{C[0, s]}=\theta \psi_{0}\left(\frac{X_{s}^{(s)}}{\sqrt{s}}\right) \quad \text { a.s. }
$$

Proposition 2.1 shows that the above limit integrates to 1 , and so for $s$ fixed $\left\{d P_{X^{(v)}} /\left.d P_{X^{(s)}}\right|_{C[0, s]} \mid v \geq s\right\}$ is uniformly integrable. Therefore $P_{X^{(v)}} \stackrel{\mathrm{w}}{\rightarrow} Q^{(s)}$ on $C[0, s]$ as $v \rightarrow \infty$, where

$$
\frac{d Q^{(s)}}{d P_{X^{(s)}}}=\theta \psi_{0}\left(\frac{X_{s}^{(s)}}{\sqrt{s}}\right)
$$

Since $s>0$ is arbitrary, $X^{(v)} \stackrel{\mathrm{w}}{\rightarrow} X^{\infty}$ on $C[0, \infty)$ as $v \rightarrow \infty$, where $P_{X^{\infty}}$ satisfies (2.8). (2.11) is now a consequence of (2.8) and the fact that $\left|X^{(s)}(u)\right|<c \sqrt{u}$ for all $0<u \leq s$ a.s.

Use (2.2) and (2.8) to see that for $\phi$ bounded and measurable

$$
E\left(\phi\left(X_{t}^{\infty}\right)\right)=E\left(\phi\left(X_{t}^{(t)}\right) \psi_{0}\left(\frac{X_{t}^{(t)}}{\sqrt{t}}\right)\right) \theta=\int_{-c}^{c} \phi\left(y t^{1 / 2}\right) \psi_{0}(y)^{2} m(d y)
$$

To establish the Markov property, fix $0<s<t$ and note that (2.7) and (2.8) imply

$$
\left.\frac{d P_{X^{\infty}}}{d P_{X^{(t)}}}\right|_{C[0, s]}=\psi_{0}\left(\frac{X_{s}^{(t)}}{\sqrt{s}}\right) \theta\left(\psi\left(s, t, X_{s}^{(t)}\right)\right)^{-1},
$$

where $\psi\left(s, v, X_{s}^{(s)}\right)$ denotes the right side of (2.7). Therefore if $\phi_{1}$ is a bounded measurable function on $C[0, s]$ and $\phi_{2}$ is a bounded measurable function on $\mathbf{R}$, then

$$
\begin{aligned}
& E\left(\phi_{1}\left(X_{u}^{\infty}, u \leq s\right) \phi_{2}\left(X_{t}^{\infty}\right)\right)=E\left(\phi_{1}\left(X_{u}^{(t)}, u \leq s\right) \phi_{2}\left(X_{t}^{(t)}\right) \psi_{0}\left(X_{t}^{(t)} / \sqrt{t}\right)\right) \theta \\
&=E\left(\phi_{1}\left(X_{u}^{(t)}, u \leq s\right) P_{s, t}^{(t)}\left(\phi_{2}(\cdot) \psi_{0}(\cdot / \sqrt{t})\right)\left(X_{s}^{(t)}\right)\right) \theta \quad \text { (Theorem 2.2) } \\
&=E\left(\phi_{1}\left(X_{u}^{\infty}, u \leq s\right) P_{s, t}^{(t)}\left(\phi_{2}(\cdot) \psi_{0}(\cdot / \sqrt{t})\right)\left(X_{s}^{\infty}\right) \theta\right. \\
&\left.\times \psi_{0}\left(X_{s}^{\infty} / \sqrt{s}\right)^{-1} \theta^{-1} \psi\left(s, t, X_{s}^{\infty}\right)\right) \quad \text { by (2.13). }
\end{aligned}
$$

This shows $X^{\infty}$ is a Markov process with transition function

$$
P_{s, t} \phi(x)=P_{s, t}^{(t)}\left(\phi(\cdot) \psi_{0}(\cdot / \sqrt{t})\right)(x) \psi_{0}(x / \sqrt{s})^{-1} \psi(s, t, x), \quad|x|<c \sqrt{s} .
$$

It is now a straightforward computation to show that the above expression equals the right side of (2.9). Since (2.10) is clear from (2.12), this completes the proof. 
We now recall some well-known results about the Ornstein-Uhlenbeck process $\tilde{Z}_{t}$, killed when it leaves $[-c, c](c>0)$. Denote this killed process by $\hat{Z}_{t}$. The semigroup of this process is denoted by

$$
\hat{P}_{t} \phi(x)=\hat{P}_{t}^{c} \phi(x)
$$

and maps $C_{0}[-c, c]$ to $C_{0}[-c, c]$, where

$$
C_{0}[-c, c]=\{\phi \in C[-c, c] \mid \phi( \pm c)=0\} \text {. }
$$

If $\hat{A}=\hat{A}^{c}$ denotes the infinitesimal generator of $\hat{P}_{t}$, then $\hat{A}=A$ on

$$
D(\hat{A})=\left\{F \in C_{0}[-c, c] \mid F^{\prime} \text { is continuous on }(-c, c) \text { and } A F \in C_{0}[-c, c]\right\} .
$$

(See Knight (1981, p. 93) and note that the continuity of $\left(d^{+} / d x\right)(d / d x) F$ for $F \in D(\hat{A})$ allows one to replace $d^{+} / d x$ with $d / d x$.)

Proposition 2.4. (a)

$$
\hat{P}_{t} \phi(x)=\int_{-c}^{c} \phi(y) \hat{p}(t, x, y) m(d y) \quad \text { for } t>0
$$

where

$$
\hat{p}(t, x, y)=\hat{p}^{c}(t, x, y)=\sum_{n=0}^{\infty} e^{-\lambda_{n} t} \psi_{n}(x) \psi_{n}(y),
$$

and the convergence in (2.14) is absolute and uniform in $(t, x, y) \in[\varepsilon, \infty) \times[-c, c]^{2}$ for each $\varepsilon>0$.

(b) For each $(t, y) \in(0, \infty) \times[-c, c], \hat{p}(t, \cdot, y) \in D(\hat{A})$ and

$$
\begin{aligned}
\hat{A}(\hat{p}(t, \cdot, y))(x) & =\frac{\partial}{\partial t} \hat{p}(t, x, y)=-\sum_{n=0}^{\infty} \lambda_{n} e^{-\lambda_{n} t} \psi_{n}(x) \psi_{n}(y), \\
\frac{\partial}{\partial x} \hat{p}(t, x, y) & =\sum_{n=0}^{\infty} e^{-\lambda_{n} t} \psi_{n}^{\prime}(x) \psi_{n}(y)
\end{aligned}
$$

where the series converge absolutely and uniformly on $[\varepsilon, \infty) \times[-c, c]^{2}$ for each $\varepsilon>0$. Moreover, $\hat{p}(\cdot, \cdot, \cdot)$ is $C^{2}$ on $(0, \infty) \times[-c, c]^{2}$.

PROOF. If

$$
\hat{p}_{N}(t, x, y)=\sum_{n=0}^{N} e^{-\lambda_{n} t} \psi_{n}(x) \psi_{n}(y),
$$

then it is well known (see e.g. Lemma $3 \mathrm{a}$ of $[\mathbf{1 0}]$ and its proof) that $\hat{p}_{N}(t, x, y)$ converges absolutely and uniformly on $[\varepsilon, \infty) \times[-c, c]^{2}$ for each $\varepsilon>0$ as $N \rightarrow \infty$ to a density, with respect to $m, \hat{p}(t, x, \cdot)$, for $\hat{P}_{t} \phi(x)$. Clearly $\hat{p}_{N}(t, \cdot, y) \in D(\hat{A})$ and

$$
\hat{A}\left(\hat{p}_{N}(t, \cdot, y)\right)(x)=-\sum_{n=0}^{N} \lambda_{n} e^{-\lambda_{n} t} \psi_{n}(x) \psi_{n}(y) .
$$

If $\varepsilon>0$, then for $t \geq \varepsilon$, large enough $M$ (depending on $\varepsilon$ ) and $N>M$,

$$
\begin{aligned}
\sum_{n=M}^{N} \lambda_{n} e^{-\lambda_{n} t} & \left|\psi_{n}(x)\right|\left|\psi_{n}(y)\right| \\
& \leq\left(\sum_{M}^{N} e^{-\lambda_{n} t / 2} \psi_{n}(x)^{2}\right)^{1 / 2}\left(\sum_{M}^{N} e^{-\lambda_{n} t / 2} \psi_{n}(y)^{2}\right)^{-1 / 2} .
\end{aligned}
$$


The right side approaches zero uniformly on $[\varepsilon, \infty) \times[-c, c]^{2}$ as $M, N \rightarrow \infty$ by (a). Since $\hat{A}$ is a closed operator, it follows that $\hat{p}(t, \cdot, y) \in D(\hat{A})$ and

$$
\hat{A}(\hat{p}(t, \cdot, y))(x)=-\sum_{n=0}^{\infty} \lambda_{n} e^{-\lambda_{n} t} \psi_{n}(x) \psi_{n}(y)=\frac{\partial}{\partial t} \hat{p}(t, x, y)
$$

The last equality follows from the absolute and uniform convergence of the series, which also shows $(\partial \hat{p} / \partial t)(t, x, y)$ is continuous on $(0, \infty) \times[-c, c]^{2}$. Note that if $\hat{p}_{z}(t, z, y)$ denotes the partial derivative of $\hat{p}$ with respect to $z$, then

$$
\begin{aligned}
\frac{1}{2} e^{z^{2} / 2} \frac{\partial}{\partial z}\left(e^{-z^{2} / 2} \hat{p}_{z}(t, z, y)\right) & =\hat{A}(\hat{p}(t, \cdot, y))(z) \\
& =\sum_{n=0}^{\infty}-\lambda_{n} e^{-\lambda_{n} t} \psi_{n}(z) \psi_{n}(y)
\end{aligned}
$$

Multiply both sides by $e^{-z^{2} / 2}$ and integrate from 0 to $x$ to obtain

$$
\begin{aligned}
& \frac{1}{2}\left(e^{-x^{2} / 2} \hat{p}_{x}(t, x, y)-\hat{p}_{x}(t, 0, y)\right)=\sum_{n=0}^{\infty} \psi_{n}(y) e^{-\lambda_{n} t} \int_{0}^{x} \hat{A} \psi_{n}(z) e^{-z^{2} / 2} d z \\
& \Rightarrow e^{-x^{2} / 2} \hat{p}_{x}(t, x, y)=\sum_{n=0}^{\infty} \psi_{n}(y) e^{-\lambda_{n} t} e^{-x^{2} / 2} \psi_{n}^{\prime}(x)
\end{aligned}
$$

Here we have used symmetry to show that $\hat{p}_{x}(t, 0, y)=\psi_{n}^{\prime}(0)=0$ and the expression for $\hat{A}$ given in (2.14a). Since the above series converges absolutely and uniformly on $[\varepsilon, \infty) \times[-c, c]^{2}$ for each $\varepsilon>0, \hat{p}_{x}$ is continuous on $(0, \infty) \times[-c, c]^{2}$ and hence so is $\hat{p}_{x x}$ by the joint continuity of $\hat{A}(\hat{p}(t, \cdot, y))(x)$. Finally, a direct differentiation of the above series expansions for $\hat{p}_{t}$ and $\hat{p}_{x}$ shows that $\hat{p}_{t t}$ and $\hat{p}_{x t}$ are also continuous on $(0, \infty) \times[-c, c]^{2}$.

Notation. (a) If $c>0, \phi \in C[-c, c], t \geq 0$ and $x \in(-c, c)$, let

$$
\tilde{P}_{t} \phi(x)=\tilde{P}_{t}^{c} \phi(x)=\hat{P}_{t}^{c}\left(\psi_{0}(c) \phi\right)(x) \psi_{0}(c)(x)^{-1} e^{\lambda_{0}(c) t}
$$

(b) Let $X^{\infty}(t, \omega)$ denote the process constructed in Theorem 2.3(b), and defined on the canonical space of continuous paths $\left(\tilde{\Omega}, \tilde{\mathcal{F}}, \tilde{F}_{t}, P_{X^{\infty}}\right)$, and let $Y^{\infty}(u)=$ $X^{\infty}\left(e^{u}\right) e^{-u / 2}$ for $u \in \mathbf{R}$. We write $P$ for $P_{X^{\infty}}$ if there is no confusion with our earlier notation.

THEOREM 2.5. (a) Fix $c>0$. If $\phi \in C[-c, c]$, $\tilde{P}_{t} \phi(\cdot)$ has a continuous extension to $[-c, c] .\left\{\tilde{P}_{t} \mid t \geq 0\right\}$ is then a strongly continuous Markov semigroup on $C[-c, c]$ with infinitesimal generator

$$
\tilde{A} F(x)=\tilde{A}^{c} F(x)=\frac{1}{2} F^{\prime \prime}(x)+\left(\psi_{0}^{\prime}(x) / \psi_{0}(x)-x / 2\right) F^{\prime}(x), \quad|x|<c,
$$

on

$$
D(\tilde{A})=\left\{F \in C[-c, c] \mid F^{\prime \prime} \text { is continuous on }(-c, c), \tilde{A} F \in C[-c, c]\right\} .
$$

$\left\{\tilde{P}_{t} \mid \geq 0\right\}$ is the semigroup of the diffusion on $(-c, c)$ with scale function

$$
\tilde{s}(x)=\tilde{s}^{c}(x)=\int_{0}^{x} e^{y^{2} / 2} \psi_{0}(c)(y)^{-2} d y
$$

and speed measure

$$
2 d \tilde{m}(x)=2 d \tilde{m}^{c}(x)=2 \psi_{0}(c)(x)^{2} d m(x) .
$$


Each of the end points $\{ \pm c\}$ is an entrance but not an exit.

(b) If $c>1, Y^{\infty}(u)$ is the stationary diffusion on $(-c, c)$ with semigroup $\tilde{P}_{t}^{c}$ and stationary measure $\tilde{m}^{c}$. More specifically, if $T$ is a finite $\left\{\mathcal{F}_{t}^{Y^{\infty}}\right\}$-stopping time, then

$$
E\left(\phi\left(Y_{T+t}^{\infty}\right) \mid \mathcal{F}_{T}^{Y^{\infty}}\right)=\tilde{P}_{t} \phi\left(Y_{T}^{\infty}\right) \quad \text { a.s. for } \phi \text { bounded measurable. }
$$

ProOF. (a) Since $\hat{p}(t, \pm c, y)=0, \partial \hat{p} / \partial x(t, x, y)$ is continuous on $(0, \infty) \times[-c, c]^{2}$ (Proposition 2.4) and $\psi_{0}^{\prime}( \pm c) \neq 0$ (or else $\psi_{0} \equiv 0$ by the classical uniqueness theorem for ODEs), an elementary calculus argument shows that

$$
\hat{p}(t, x, y) / \psi_{0}(x) \text { has a continuous extension to }(0, \infty) \times[-c, c]^{2} .
$$

Therefore if $\phi \in C[-c, c]$,

$$
\tilde{P}_{t} \phi(x)=\int_{-c}^{c} \frac{\hat{p}(t, x, y) \psi_{0}(y) \phi(y) m(d y) e^{\lambda_{0} t}}{\psi_{0}(x)}
$$

has a continuous extension to $[-c, c]$. Proposition 2.4 shows that $\hat{P}_{t} \psi_{0}=e^{-\lambda_{0} t} \psi_{0}$, and hence $\tilde{P}_{t}$ is a Markov semigroup on $C[-c, c]$. To prove the strong continuity of $\left\{\tilde{P}_{t}\right\}$, take $F \in C^{2}[-c, c]$ such that $F^{\prime}( \pm c)=0$ and show there is uniform convergence in $x$ as $t \downarrow 0$ in (2.17) below.

Let $F \in D(\tilde{A})$. Since

$$
\begin{aligned}
\left(\tilde{P}_{t} F(x)-F(x)\right) t^{-1}= & \psi_{0}(x)^{-1} e^{\lambda_{0} t}\left(\hat{P}_{t}\left(F \psi_{0}\right)(x)-F \psi_{0}(x)\right) t^{-1} \\
& +\left(e^{\lambda_{0} t}-1\right) t^{-1} F(x)
\end{aligned}
$$

clearly $F \psi_{0} \in D(\hat{A})$. This implies $F^{\prime \prime}$ is continuous on $(-c, c)$ and allows us to obtain

$$
\begin{aligned}
\left(\tilde{P}_{t} F\right. & (x)-F(x)) t^{-1} \\
& =\psi_{0}(x)^{-1} e^{\lambda_{0} t} \int_{0}^{t} \hat{P}_{u}\left(\hat{A}\left(F \psi_{0}\right)\right)(x) d u t^{-1}+\left(e^{\lambda_{0} t}-1\right) t^{-1} F(x) \\
& \rightarrow \psi_{0}(x)^{-1} \hat{A}\left(F \psi_{0}\right)(x)+\lambda_{0} F(x), \quad \text { as } t \rightarrow 0+, \forall x \in(-c, c) \\
& =A F(x)+\frac{\psi_{0}^{\prime}(x)}{\psi_{0}(x)} F^{\prime}(x) \equiv A_{0} F(x)
\end{aligned}
$$

Therefore

$$
\begin{aligned}
& D(\tilde{A}) \subset\left\{F \in C[-c, c] \mid F^{\prime \prime}\right. \text { is continuous } \\
& \left.\quad \text { on }[-c, c] \text { and } A_{0} F \in C[-c, c]\right\} \equiv D_{0},
\end{aligned}
$$

and $\tilde{A}=A_{0}$ on $D_{0}$. Since $A_{0}$ with domain $D_{0}$ is the generator of the diffusion with scale function and speed measure $\tilde{s}$ and $2 \tilde{m}$, respectively (see Knight (1981, pp. 91-92), but note we may change the right derivatives to derivatives in this case), it follows from Williams $[1979$, p. 113, Lemma (5)] that there is equality in (2.18). The classification of the endpoints follows from Knight (1981, p. 182).

(b) (2.11) implies $Y_{u}^{\infty} \in(-c, c)$ for all $u \geq 0$ a.s., and an easy computation using (2.9) shows that if $\phi$ is bounded and measurable, then

$$
E\left(\phi\left(Y_{t}^{\infty}\right) \mid Y_{u}^{\infty} \leq s\right)=\tilde{P}_{t-s} \phi\left(Y_{s}^{\infty}\right) \quad \text { a.s. } \forall-\infty<s<t .
$$


Since $\tilde{P}_{t}: C[-c, c] \rightarrow C[-c, c],(2.15)$ follows from the usual proof of the strong Markov property for Feller processes. Finally, it is clear from $(2.10)$ that $Y^{\infty}(u)$ has law $\tilde{m}$ and hence that $Y^{\infty}$ is a stationary diffusion.

If $c>1$ is fixed, let

$$
\tilde{Y}=\left(\tilde{\Omega}, \tilde{\mathcal{F}}, \tilde{\mathcal{F}}_{t}, \tilde{Y}_{t}, \theta_{t}, \tilde{P}^{x}\right), \quad x \in[-c, c]
$$

be the diffusion with semigroup $\left\{\tilde{P}_{t}\right\}$ and defined on the canonical space of paths, $C[0, \infty)=\tilde{\Omega}$. Since $\pm c$ are entrances but not exits, we have

$$
\tilde{P}^{ \pm c}(\tilde{Y}(0)= \pm c)=1 \quad \text { and } \quad \tilde{P}^{ \pm c}(|\tilde{Y}(t)|<c \text { for all } t>0)=1 .
$$

Moreover, it is clear from Theorem $2.5($ a) that $\tilde{P} \stackrel{\text { w }}{\rightarrow} \tilde{P} \quad$ as $x \rightarrow \pm c . \tilde{P} \quad$ is the law of $\left\{Y^{\infty}(u) \mid u \geq 0\right\}$, and it follows easily from the definition of $\left\{\tilde{P}_{t}\right\}$ that for any $\tilde{\mathcal{F}}_{t}$-measurable random variable $R: \tilde{\Omega} \rightarrow[0, \infty)$,

$$
\tilde{E}^{x}(R)=E^{x}\left(R(\tilde{Z}) \psi_{0}\left(\tilde{Z}_{t}\right) I(\rho>t)\right) \psi_{0}(x)^{-1} e^{\lambda_{0} t}, \quad x \in(-c, c) .
$$

Note that if $\tilde{Y}$ is killed at an independent exponential time with mean $\lambda_{0}^{-1}$, the resulting process would be the $h$-path process obtained from $Z$, where $h$ is the positive excessive function (for $\hat{Z}), \psi_{0}$ [see Doob $(1984$, p. 566)]. Hence, " $\tilde{Y}$ is $\tilde{Z}$ conditioned not to hit $\pm c$ ".

It is now a routine exercise to show that $X^{\infty}$ and $Y^{\infty}$ are solutions of the appropriate stochastic differential equations. If

$$
g(y)=-y / 2+\psi_{0}^{\prime}(y) / \psi_{0}(y)
$$

then

$$
E\left(\int_{s}^{t}\left|g\left(Y_{u}^{\infty}\right)\right| d u\right)=(t-s) \int_{-c}^{c}|g(y)| \psi_{0}^{2}(y) m(d y)<\infty
$$

If $-\infty<s<t<\infty$, let

$$
W(s, t]=Y^{\infty}(t)-Y^{\infty}(s)-\int_{s}^{t} g\left(Y_{u}^{\infty}\right) d u .
$$

Although $f(x)=x \notin D(\tilde{A})$, an obvious pointwise approximation of $f$ and its first two derivatives by functions in $C^{2}[-c, c]$ whose first derivatives vanish at $\pm c$ allows one to apply Dynkin's formula to $f$ to get

$$
\begin{aligned}
E\left(Y_{t}^{\infty}-Y_{s}^{\infty} \mid \mathcal{F}_{s}^{Y^{\infty}}\right) & =\tilde{P}_{t-s} f\left(Y_{s}^{\infty}\right)-Y_{s}^{\infty} \\
& =\tilde{E}^{Y_{s}^{\infty}}\left(\int_{0}^{t-s} g\left(\tilde{Y}_{u}\right) d u\right)=E\left(\int_{s}^{t} g\left(Y_{u}^{\infty}\right) d u \mid \mathcal{F}_{s}^{Y^{\infty}}\right)
\end{aligned}
$$

whence

$$
E\left(W(s, t] \mid \xi_{s}^{Y^{\infty}}\right)=0 \text { for }-\infty<s<t<\infty
$$


Also, for $s<t$,

$$
\begin{aligned}
& E\left(W(s, t]^{2} \mid \xi_{s}^{Y^{\infty}}\right)=E\left(\left(Y^{\infty}(t)-\int_{s}^{t} g\left(Y_{u}^{\infty}\right) d u\right)^{2}-Y^{\infty}(s)^{2} \mid \xi_{s}^{Y^{\infty}}\right) \quad \text { (use (2.21) twice) } \\
&= \tilde{E}^{Y_{s}^{\infty}}\left(\tilde{Y}_{t-s}^{2}-\tilde{Y}_{0}^{2}-2 \int_{0}^{t-s} \tilde{Y}_{t-s} g\left(\tilde{Y}_{u}\right) d u+\left(\int_{0}^{t-s} g\left(\tilde{Y}_{u}\right) d u\right)^{2}\right) \\
&= \tilde{E}^{Y_{s}^{\infty}}\left(\int_{0}^{t-s} \tilde{A} h\left(\tilde{Y}_{u}\right) d u\right)-2 \tilde{E}^{Y_{s}^{\infty}}\left(\int_{0}^{t-s}\left(\tilde{E}^{\tilde{Y}(u)}\left(\tilde{Y}_{t-s-u}\right)\right) g\left(\tilde{Y}_{u}\right) d u\right) \\
&+\tilde{E}^{Y_{s}^{\infty}}\left(\left(\int_{0}^{t-s} g\left(\tilde{Y}_{u}\right) d u\right)^{2}\right),
\end{aligned}
$$

where $h(x)=x^{2}$, and the application of Dynkin's formula is again justified by approximating $h$ by functions in $D(\tilde{A})$. By $(2.21)$ the above equals

$$
\begin{aligned}
(t-s) & +2 \tilde{E}^{Y_{s}^{\infty}} \int_{0}^{t-s} \tilde{Y}_{u} g\left(\tilde{Y}_{u}\right) d u-2 \tilde{E}^{Y_{s}^{\infty}} \int_{0}^{t-s} \tilde{Y}_{u} g\left(\tilde{Y}_{u}\right) d u \\
& -2 \tilde{E}^{Y_{s}^{\infty}}\left(\int_{0}^{t-s} \tilde{E}^{\tilde{Y}_{u}}\left(\int_{0}^{t-s-u} g\left(\tilde{Y}_{w}\right) d w\right) g\left(\tilde{Y}_{u}\right) d u\right) \\
& +\tilde{E}^{Y_{s}^{\infty}}\left(\left(\int_{0}^{t-s} g\left(\tilde{Y}_{u}\right) d u\right)^{2}\right)=t-s .
\end{aligned}
$$

It follows that $W$ is an $\left\{\mathcal{F}_{t}^{Y^{\infty}}\right\}$-white noise on $\mathbf{R}$ (with respect to Lebesgue measure). Hence, $Y^{\infty}$ is a solution of

$$
\begin{aligned}
Y_{t}^{\infty}-Y_{s}^{\infty}=W(s, t]+\int_{s}^{t} g\left(Y_{u}^{\infty}\right) d u & \text { for }-\infty<s<t<\infty, \\
& \text { where } g(y)=-y / 2+\psi_{0}^{\prime}(y) / \psi_{0}(y) .
\end{aligned}
$$

$\hat{W}(s, t]=W(\log s, \log t]$ defines an $\left\{\hat{f}_{t}\right\}$-white noise on $(0, \infty)$ with respect to the measure $\nu(A)=\int_{A} t^{-1} d t$. If

$$
\beta_{t}=\int_{(0, t]} u^{1 / 2} d \hat{W}(u),
$$

then it is easy to see that $\beta$ is on $\left\{\tilde{\mathcal{F}}_{t}\right\}$-Brownian motion. For $s>0$ fixed, $\left\{Y^{\infty}(\log t) \mid t \geq s\right\}$ is a semimartingale by (2.22), and so an integration by parts gives

$$
\begin{aligned}
X_{t}^{\infty}-X_{s}^{\infty}= & t^{1 / 2} Y^{\infty}(\log t)-s^{1 / 2} Y^{\infty}(\log s) \\
= & \int_{s}^{t} Y^{\infty}(\log u) \frac{1}{2} u^{-1 / 2} d u+\int_{s}^{t} u^{1 / 2} d\left(Y^{\infty}(\log u)\right) \\
= & \int_{s}^{t} X^{\infty}(u)(2 u)^{-1} d u+\beta_{t}-\beta_{s} \\
& +\int_{s}^{t}\left(\frac{\psi_{0}^{\prime}}{\psi_{0}}\left(Y^{\infty}(\log u)\right)-\frac{1}{2} Y^{\infty}(\log u)\right) u^{1 / 2} u^{-1} d u
\end{aligned}
$$




$$
X_{t}^{\infty}-X_{s}^{\infty}=\beta_{t}-\beta_{s}+\int_{s}^{t} \frac{\psi_{0}^{\prime}}{\psi_{0}}\left(\frac{X_{u}^{\infty}}{\sqrt{u}}\right) \frac{d u}{\sqrt{u}} .
$$

As in the proof of $(2.20)$, ones gets

$$
E\left(\int_{0}^{t}\left|\frac{\psi_{0}^{\prime}}{\psi_{0}}\left(\frac{X_{u}^{\infty}}{\sqrt{u}}\right)\right| \frac{d u}{\sqrt{u}}\right)<\infty \quad \forall t>0
$$

and hence we may let $s \downarrow 0$ in (2.23) and conclude that

$$
X_{t}^{\infty}=\beta_{t}+\int_{0}^{t} \frac{\psi_{0}^{\prime}}{\psi_{0}}\left(\frac{X_{u}^{\infty}}{\sqrt{u}}\right) \frac{d u}{\sqrt{u}}, \quad t \geq 0 .
$$

In particular, (2.24) and (2.25) show $X^{\infty}$ is a semimartingale. In fact it is not hard to show that equations (2.22) and (2.25) uniquely determine $Y^{\infty}$ and $X^{\infty}$, respectively.

THEOREM 2.6. (a) If $W$ is a white noise on $\mathbf{R}$ (with respect to Lebesgue measure), there is a unique solution $\left\{Y_{u}^{\infty} \mid u \in \mathbf{R}\right\}$ of (2.22). The solution has the same law as the stationary diffusion described in Theorem 2.5(b).

(b) If $\beta_{t}$ is a Brownian motion $\left(\beta_{0}=0\right)$, there is a unique solution $\left\{X_{t}^{\infty} \mid t \geq 0\right\}$ of (2.25). The solution has the same law as the inhomogeneous diffusion described in Theorem 2.3(b).

We need a result that follows from Kunita [26, Theorem 5.2] and Yamada and Ogura [25, Theorem 3.1]. $\Delta$ is added to $\mathbf{R}$ as the point at $\infty$.

THEOREM 2.7. Let $\sigma, b:(-c, c) \rightarrow \mathbf{R}$. Assume there are constants $\left\{K_{n}\right\}$ such that

$$
|\sigma(x)-\sigma(y)|+|b(x)-b(y)| \leq K_{n}|x-y| \quad \text { for } x, y \in\left(-c+n^{-1}, c-n^{-1}\right) .
$$

Let $\beta$ be an $\mathcal{F}_{t}^{0}$-Brownian motion on $\left(\Omega^{0}, \mathcal{F}^{0}, \mathcal{F}_{t}^{0}, P^{0}\right)$. There are measurable mappings $X:[0, \infty) \times(-c, c) \times \Omega \rightarrow \mathbf{R} \cup\{\Delta\}, T:(-c, c) \times \Omega \rightarrow[0, \infty]$ such that

(a) For each $x$ in $(-c, c), X_{t}(x)$ is an $\left\{\mathcal{F}_{t}^{0}\right\}$-adapted solution of

$$
\begin{aligned}
& X_{t}=\left\{\begin{array}{l}
x+\int_{0}^{t} \sigma\left(X_{s}\right) d \beta_{s}+\int_{0}^{t} b\left(X_{s}\right) d s, \quad t<T(x) \text { a.s. } \\
\Delta, \quad t \geq T(x),
\end{array}\right. \\
& \lim _{t \uparrow T(x)}\left|X_{t}(x)\right|=c \quad \text { if } T(x)<\infty \text { a.s. }
\end{aligned}
$$

If $X^{\prime}, T^{\prime}$ also satisfy the above ( $x$ fixed), then $X^{\prime}=X(x), T^{\prime}=T(x)$ a.s.

(b) $T(\cdot)$ is lower semicontinuous and $X(\cdot, \cdot)(\omega)$ is continuous on the open set $\{(t, x) \mid t<T(x)\}$ a.s.

(c) For a.a. $\omega$, if $-c<x<y<c$ and $t<T(x) \wedge T(y)$, then $X(t, x)<X(t, y)$.

Although these results are stated in the above references for $c=\infty$, they follow on a finite interval by mapping $\mathbf{R}$ diffeomorphically onto $(-c, c)$. The pathwise uniqueness in (2.26) allows one to apply the method of Yamada and Watanabe [23] to conclude that uniqueness in law holds in (2.26) as well.

PROOF OF THEOREM 2.6. (a) Apply the above result with $\sigma=1, b=g, \beta_{t}=$ $W(-n,-n+t]$ to obtain $X_{n}(t, x)=X(t+n, x)$ and $T_{n}(x)=T(x)-n$, where $X$ and $T$ are as in the theorem, satisfying

$$
X_{n}(t, x)=x+W(-n, t]+\int_{-n}^{t} g\left(X_{n}(x, s)\right) d s, \quad-n \leq t<T_{n}(x) .
$$


The arguments leading up to (2.22) and uniqueness in law in (2.27) show that $t \rightarrow X_{n}(-n+t, x)$ has law $\tilde{P}^{x}$. In particular, $T_{n}(x)=\infty$ a.s. for each $x$, and therefore $T_{n}(x)=\infty$ for all $x$ a.s. (see Kunita [26, p. 70]). The monotonicity of $X_{n}(t, \cdot)$ allows us to define

$$
X_{n}(t, \pm c)=\lim _{\substack{x \rightarrow \pm c \\|x|<c}} X_{n}(t, x) \in[-c, c], \quad t \geq-n \text { a.s }
$$

The law of $X_{n}(-n+t, \pm c)$ is $\tilde{P}^{ \pm c}$ because $\tilde{P}^{x} \stackrel{\mathrm{w}}{\rightarrow} \tilde{P}^{ \pm c}$ as $x \rightarrow \pm c$, and, in particular, $\left|X_{n}(t, \pm c)\right|<c$ for all $t>-n$ a.s. Note that $g\left(X_{n}(s, x)\right) \downarrow g\left(X_{n}(s, c)\right)$ as $x \uparrow c$ by the monotonicity of $g$ (Lemma 4.3(b)). Use the monotone convergence theorem to let $x \uparrow c$ in (2.27) and get

$$
X_{n}(t, c)=c+W(-n, t]+\int_{-n}^{t} g\left(X_{n}(s, c)\right) d s, \quad-n \leq t \text { a.s. }
$$

If $m>n, X_{m}(t, c)=X_{n}\left(t, X_{m}(-n, c)\right)$ for all $t \geq-n$ a.s. by pathwise uniqueness in

$$
X_{t}=X_{m}(-n, c)+W(-n, t]+\int_{-n}^{t} g\left(X_{s}\right) d s, \quad\left|X_{t}\right|<c, t>-n .
$$

The strict monotonicity of $X_{n}(t, \cdot)$ therefore shows that for a.a. $\omega, m>n, t>-n$,

$$
-c<X_{n}(t,-c)<X_{m}(t,-c)<X_{m}(t, c)<X_{n}(t, c)<c,
$$

and we may define

$$
X_{+}(t)=\lim _{m \rightarrow \infty} X_{m}(t, c) \in(-c, c), \quad t \in \mathbf{R},
$$

where the limit is strictly decreasing and in $(-c, c)$ by $(2.29)$. Let $n \rightarrow \infty$ in (2.28) and use monotone convergence again to see that $X_{+}$is a solution of (2.22). If $Y$ is any solution of (2.22), then pathwise uniqueness in (2.27) shows that

$$
Y(t)=X_{n}\left(t, Y_{-n}\right) \leq X_{n}(t, c) \text { for } t \geq-n \text { a.s. }
$$

Let $n \rightarrow \infty$ to obtain $Y(t) \leq X_{+}(t)$ for all $t \in \mathbf{R}$ a.s. Similarly one can construct a solution to (2.22), $X_{-}$, such that $X_{-}(t) \leq Y(t)$ for all $t \in \mathbf{R}$ a.s. and for any solution $Y$ of (2.22). Pathwise uniqueness would now follow if we show the law of $Y_{t}$ is $\tilde{m}$ for any solution $Y$, as this would force $X_{-}(t)=X_{+}(t)$ a.s. Fix $t \in \mathbf{R}$ and such a $Y$. If $\mu_{n}$ is the law of $Y(-n)$, then (2.30) shows that for $t>-n$,

$$
P\left(Y_{t} \in A\right)=\int_{-c}^{c} \int_{A} \hat{p}(t+n, x, y) \psi_{0}(x)^{-1} \psi_{0}(y) d m(y) e^{\lambda_{0} t} d \mu_{n}(x) .
$$

If

$$
r(t, x, y)=\sum_{n=1}^{\infty} e^{-\left(\lambda_{n}-\lambda_{1}\right) t} \psi_{n}(x) \psi_{n}(y),
$$

then (2.14) shows that for $s \geq 1$,

$$
\begin{gathered}
\left|\hat{p}(s, x, y) \psi_{0}(x)^{-1} e^{\lambda_{0} s}-\psi_{0}(y)\right| \leq e^{-\left(\lambda_{1}-\lambda_{0}\right) s}|r(s, x, y)| \psi_{0}(x)^{-1} \\
\leq e^{-\left(\lambda_{1}-\lambda_{0}\right) s}\left(\sum_{n=1}^{\infty} e^{-\left(\lambda_{n}-\lambda_{1}\right) s} \psi_{n}(x)^{2}\right)^{1 / 2} \\
\quad \times\left(\sum_{n=1}^{\infty} e^{-\left(\lambda_{n}-\lambda_{1}\right) s} \psi_{n}(y)^{2}\right)^{1 / 2} \psi_{0}(x)^{-1} \\
\leq e^{\lambda_{1}} e^{-\left(\lambda_{1}-\lambda_{0}\right) s}\left(\hat{p}(1, x, x) \psi_{0}(x)^{-2}\right)^{1 / 2} \hat{p}(1, y, y)^{1 / 2}
\end{gathered}
$$


Proposition 2.4(b) implies $f(x)=\hat{p}(1, x, x)$ is $C^{2}$ on $[-c, c]$ and $f^{\prime}( \pm c)=0$, which in turn implies that $\hat{p}(1, x, x) \psi_{0}(x)^{-2}$ is uniformly bounded on $(-c, c)$. Therefore there is a constant $K$ such that $(2.32)$ is bounded by $K e^{-\left(\lambda_{1}-\lambda_{0}\right) s}$. Let $s=t+n$, substitute this bound into (2.31) and let $n \rightarrow \infty$ to see that $P\left(Y_{t} \in A\right)=\tilde{m}(A)$. (We have used the fact that $\mu_{n}((-c, c))=1-$ see (2.30).) This completes the proof of (a).

(b) As above, it remains only to prove pathwise uniqueness in (2.25). Before stating Theorem 2.6, we showed that if $Y$ is a solution of $(2.22)$, then $X_{t}=t^{1 / 2} Y(\log t)$ is a solution of (2.25). It is just as easy to go in the opposite direction. This correspondence and (a) give the result.

All the results of this section remain valid (with the obvious changes) if $[-c, c]$ is replaced with a finite interval $\left[c_{1}, c_{2}\right]$ containing zero. In fact one may take $c_{1}=-\infty$ or $c_{2}=+\infty$ in Theorems 2.2 and 2.3. If $\left[c_{1}, c_{2}\right]=[0, \infty)$, then $X^{(v)}$ is the Brownian meander on $[0, v]$ [see Iglehart (1974)].

3. A stochastic differential equation for Brownian motion at a slow point. Throughout this section we work in the filtered space $\left(\Omega, \mathcal{F}_{,} \mathcal{F}_{t}, P\right)$ described in $\S 1$ and use the notation introduced there. We will show that the Brownian path at a slow point, i.e. $X^{(v)}$, is a semimartingale and in fact satisfies a stochastic differential equation analogous to (2.25). Instead of deriving this equation from Theorem 2.2, we use a "grossissement d'une filtration" because it is a more "concrete" method, involving only processes on $(\Omega, \mathcal{F})$, and was the way the equation was originally found.

We fix $v>0$ and usually suppress the dependence on $v$ in our notation. The definitions of $S=S_{v}$ and $A$ (as in (1.2d)) in $\S 1$ imply that

$$
R \equiv S+v=\inf \{t \mid A(t)>v\}
$$

is an $\left\{\mathcal{F}_{t}\right\}$-stopping time. If $s<t$, then

$$
\{S \leq s\}=\{S \leq t\} \cap F_{s t} \text { a.s. }
$$

where

$$
F_{s t}=\left\{A_{t} \geq t-s\right\} \cup\left\{A_{t}<t-s, \sup _{u \leq \tau_{s}^{+} \wedge t} A_{u}>v\right\} \in \mathcal{F}_{t} .
$$

Therefore $S$ is an honest time in the sense of Meyer, Smythe and Walsh (1972) or Barlow (1978), and we can use an enlarged filtration to study $X^{(v)}$. If $\left\{\mathcal{G}_{t}\right\}$ is the smallest filtration, satisfying the usual conditions, that contains $\left\{\mathcal{F}_{t}\right\}$ and for which $S$ is a stopping time, then $X_{t}^{(v)}$ is an $\left\{\mathscr{H}_{t}\right\}$-semimartingale where $\mathscr{H}_{t}=\mathcal{G}_{S+t}$ [see Barlow (1978, Theorem 3.10)].

Let $C_{t}=I(t \geq S)$, and let ${ }^{1} C$ and $C^{1}$ denote the optional and dual optional projections of $C$ (with respect to $\left\{\xi_{t}\right\}$ ), respectively. Then $M_{t}={ }^{1} C_{t}-C_{t}^{1}$ is a square integrable $($ at $\infty)\left\{\mathcal{F}_{t}\right\}$-martingale, and [see e.g. Barlow (1978, Theorem A)]

$$
W_{t}=X_{t}^{(v)}-\int_{S}^{S+t}\left({ }^{1} C_{u-}\right)^{-1} d\langle B, M\rangle_{u}
$$

is a square integrable (up to finite times) $\left\{\mathcal{H}_{t}\right\}$-martingale. Here $\langle B, M\rangle$ is computed with respect to $\left\{\xi_{t}\right\}$. The second term has continuous sample paths of bounded variation on compacts. Therefore $[W]_{t}=t$, and $W$ must be an $\left\{H_{t}\right\}$-Brownian motion. We must now compute ${ }^{1} C$ and $\langle B, M\rangle$. 
Let

$$
h(t, x)=P^{x}(\rho(c)>t)=\int_{-c}^{c} \hat{p}(t, x, y) m(d y) .
$$

LEMMA 3.1. (a) $h$ has continuous second-order partial derivatives on $(0, \infty) \times$ $[-c, c]$.

(b) For each $t>0, h(t, \cdot)$ is increasing on $[-c, 0]$ and decreasing on $[0, c]$.

Proof. (a) Differentiating inside the integral in (3.2), we see from Proposition 2.4 (b) that $h$ is $C^{2}$ on $(0, \infty) \times[-c, c]$.

(b) The strong Markov property shows that for $0 \leq x_{1}<x_{2}<c$,

$$
\begin{aligned}
P^{x_{1}}(\rho(c) \leq v) & =E^{x_{1}}\left(I\left(\rho\left(x_{2}\right)(\omega)<v\right) E^{\tilde{Z}\left(\rho\left(x_{2}\right)(\omega)\right)}\left(\rho(c) \leq v-\rho\left(x_{2}\right)(\omega)\right)\right) \\
& \leq P^{x_{1}}\left(\rho\left(x_{2}\right)<v\right) P^{x_{2}}(\rho(c) \leq v) \\
& <P^{x_{2}}(\rho(c) \leq v) .
\end{aligned}
$$

It follows that $h(t, \cdot)$ is decreasing on $[0, c]$ and by symmetry is increasing on $[-c, 0]$.

LEMMA 3.2 .

$$
{ }^{1} C_{t}=h\left(\log (v / A(t \wedge R)), Y(t \wedge R) A(t \wedge R)^{-1 / 2}\right) I(A(t \wedge R)>0) .
$$

Here $Y$ is as in $(1.2 \mathrm{~d})$.

PROOF. Since $R$ is an $\left\{\mathcal{F}_{t}\right\}$-stopping time, the right side is clearly optional. Let $T$ be an $\left\{\mathcal{F}_{t}\right\}$-stopping time. Then $\{T>R\} \in \mathcal{F}_{T}$, and so

$$
\begin{aligned}
P\left(T \geq S \mid \mathcal{F}_{T}\right) I(T>R) & =I(T>R) \\
& =h\left(0, Y(R) A(R)^{-1 / 2}\right) I(A(R)>0) I(T>R) \quad \text { a.s. }
\end{aligned}
$$

Checking $\{T \leq R\}$, we get for a.a. $\omega$,

$$
\begin{aligned}
P\left(T \geq S \mid \mathcal{F}_{T}\right\} I(T \leq R)(\omega) & \\
= & P\left(\left|B_{t}-B_{\tau_{T}^{-}}\right| \leq C\left(t-\tau_{T}^{-}\right)^{1 / 2} \forall t \in\left[T, \tau_{T}^{-}+v\right] \mid \mathcal{F}_{T}\right) I(T \leq R)(\omega) \\
= & P^{B(T)-B\left(\tau_{T}^{-}\right)(\omega)}\left(\left|\tilde{B}_{s}\right| \leq c\left(s+A_{T}(\omega)\right)^{1 / 2} \forall s \in\left[0, v-A_{T}(\omega)\right]\right) \\
& \times I(T \leq R)(\omega) \\
= & P^{Y(T) A(T)^{-1 / 2}(\omega)}\left(\left|\tilde{B}_{s}\right| \leq c(s+1)^{1 / 2} \forall s \in\left[0, v A_{T}(\omega)^{-1}-1\right]\right) \\
& \times I\left(A_{T}>0, T \leq R\right)(\omega) \\
= & h\left(\log \left(v / A_{T}\right), Y(T) A(T)^{-1 / 2}\right)(\omega) I\left(A_{T}>0, T \leq R\right)(\omega) .
\end{aligned}
$$

This together with $(3.2 \mathrm{a})$ implies the result.

A formal application of Itô's formula yields

$$
\langle B, M\rangle_{t}=\int_{0}^{t \wedge R} h_{x}\left(\log \left(\frac{v}{A_{s}}\right), Y_{s} A_{s}^{-1 / 2}\right) A_{s}^{-1 / 2} d s
$$

This is true, but a rigorous derivation of the above poses a few technical difficulties because $Y$ is not an $\left\{\mathcal{F}_{t}\right\}$-semimartingale if $\lambda_{0}(c) \geq 1 / 2$. For our purposes it suffices to show that

$$
\langle B, M\rangle_{S+t}-\langle B, M\rangle_{S}=\int_{0}^{t \wedge v} h_{x}\left(\log \frac{v}{s}, \frac{X_{s}}{\sqrt{s}}\right) s^{-1 / 2} d s
$$


PROOF OF (3.3). Let

$$
D_{t}={ }^{1} C_{S+t}-{ }^{1} C_{S}=M_{S+t}-M_{S}+\left(C_{S+t}^{1}-C_{S}^{1}\right) .
$$

Then $D$ is an $\left\{\not_{t}\right\}$-semimartingale and, by Lemma 3.2,

$$
D_{t}=h\left(\log (v /(t \wedge v)), X(t \wedge v)(t \wedge v)^{-1 / 2}\right) .
$$

If $0<t<v$, choose $\delta \in(0, t)$ and use Itô's formula to conclude

$$
\begin{aligned}
D_{t}= & D_{\delta}+\int_{\delta}^{t}\left(\frac{1}{2} h_{x x}\left(\log \frac{v}{s}, \frac{X_{s}}{\sqrt{s}}\right)-h_{s}\left(\log \frac{v}{s}, \frac{X_{s}}{\sqrt{s}}\right)\right) s^{-1} d s \\
& +\int_{\delta}^{t} h_{x}\left(\log \frac{v}{s}, \frac{X_{s}}{\sqrt{s}}\right) s^{-1 / 2} d X_{s} .
\end{aligned}
$$

Recall that $\langle B, M\rangle=[B, M]$ is independent of the filtration, so for $t$ as above,

$$
\begin{aligned}
\langle B, M\rangle_{S+t}-\langle B, M\rangle_{S} & =[X, D]_{t} \\
& =[X, D]_{\delta}+\int_{\delta}^{t} h_{x}\left(\log \frac{v}{s}, \frac{X_{s}}{\sqrt{s}}\right) s^{-1 / 2} d[X, X]_{s} \\
& =[X, D]_{\delta}+\int_{\delta}^{t} h_{x}\left(\log \frac{v}{s}, \frac{X_{s}}{\sqrt{s}}\right) s^{-1 / 2} d s
\end{aligned}
$$

Therefore, if $|[X, D]|(s)$ denotes the total variation of $[X, D]$ on $[0, s]$, then

$$
\begin{aligned}
\int_{0}^{v}\left|h_{x}\left(\log \frac{v}{s}, \frac{X_{s}}{\sqrt{s}}\right)\right| s^{-1 / 2} d s & =\lim _{\delta \rightarrow 0^{+}}|[X, D]|(v-\delta)-\|[X, D] \mid(\delta) \\
& =\|[X, D] \mid(v)<\infty \quad \text { a.s. }
\end{aligned}
$$

Let $\delta \rightarrow 0+$ in (3.6) and conclude that

$$
\langle B, M\rangle_{S+t}-\langle B, M\rangle_{S}=\int_{0}^{t} h_{x}\left(\log \frac{v}{s}, \frac{X_{s}}{\sqrt{s}}\right) s^{-1 / 2} d s
$$

for $0<t<v$ and hence for $0 \leq t \leq v$. (3.4) shows that

$$
\langle B, M\rangle_{S+t}-\langle B, M\rangle_{S}=[X, D]_{t}=[X, D]_{t \wedge v}=\langle B, M\rangle_{S+(t \wedge v)}-\langle B, M\rangle_{S},
$$

and (3.3) now follows from the above.

It is now an easy matter to deduce the main result of this section.

THEOREM 3.3. There is an $\left\{\mathcal{H}_{t}\right\}$-Brownian motion, $W_{t}$, such that

$$
X_{t}^{(v)}=W_{t}+\int_{0}^{t \wedge v} \frac{h_{x}}{h}\left(\log \frac{v}{u}, \frac{X_{u}^{(v)}}{\sqrt{u}}\right) \frac{d u}{\sqrt{u}} \quad \forall t \geq 0 .
$$

ProOF. If $W_{t}$ is the $\left\{\mathcal{H}_{t}\right\}$-Brownian motion defined by (3.1), then (3.1), (3.3) and Lemma 3.2 imply the above equality.

Notation. If $r \geq 0$ and $v>0$, let

$$
\begin{aligned}
& S^{r, v}=\inf \left\{t \geq \tau_{r} \mid A(t+v)>v\right\} \\
& X_{t}^{r, v}=B\left(t+S^{r, v}\right)-B\left(S^{r, v}\right) .
\end{aligned}
$$

$\left\{\mathcal{G}_{t}^{r, v}\right\}$ denotes the smallest filtration, satifying the usual conditions, that contains $\left\{\mathcal{F}_{t}\right\}$ and for which $S^{r, v}$ is a stopping time.

$$
\mathcal{H}_{t}^{r, v}=\mathcal{G}_{S^{r, v}+t}^{r, v} \text {. }
$$


REMARK 3.4. It is easy to see that $X^{r, v}$ and $X^{v}$ have the same law. Indeed, the strong Markov property of $(A, Y)$ (see $(1.2 \mathrm{~d}))$ shows that $\left(A_{t}^{r}, Y_{t}^{r}\right) \equiv$ $\left(A\left(t+\tau_{r}\right), Y\left(t+\tau_{r}\right)\right)$ is equal in law to $(A, Y)$. Now note that, since $\mathcal{F}_{t}^{(B, L)}=\mathcal{F}_{t}^{(A, Y)}$, there is a measurable map $\phi^{(v)}: D\left([0, \infty), \mathbf{R}^{2}\right) \rightarrow D([0, \infty), \mathbf{R})$ such that

$$
X^{(v)}=\phi^{(v)}(A, Y), \quad X^{r, v}=\phi^{(v)}\left(A^{r}, Y^{r}\right) \quad \text { a.s. }
$$

(See the proof of Theorem 13 in Greenwood and Perkins (1983) for an explicit construction of such a $\phi^{(v)}$.)

By making only minor changes in the above arguments, one can show

THEOREM 3.5. There is an $\left\{\mathcal{H}_{t}^{r, v}\right\}$-Brownian motion, $W^{r, v}$, such that

$$
X_{t}^{r, v}=W_{t}^{r, v}+\int_{0}^{t \wedge v} \frac{h_{x}}{h}\left(\log \frac{v}{u}, \frac{X_{u}^{r, v}}{\sqrt{u}}\right) \frac{d u}{\sqrt{u}} \quad \forall t \geq 0 .
$$

4. Local time at a slow point. We now consider the behaviour of Brownian local time at a slow point and, in particular, prove Theorem 1.2. It will be easier to study the local time of the continuous semimartingale $X^{\infty}$, and therefore for most of this section our setting will be the canonical space $(\tilde{\Omega}, \tilde{\mathcal{F}}, \tilde{\mathcal{F}}, P)$ introduced before Theorem 2.5. As usual, $c$ denotes a fixed constant greater than one. The decomposition (2.25) and Yor (1978) show that $X^{\infty}$ has a jointly continuous local time given by

$$
L_{t}^{x}\left(X^{\infty}\right)=\lim _{\varepsilon \rightarrow 0+} \int_{0}^{t} I\left(X^{\infty}(s) \in[x, x+\varepsilon]\right) d s \varepsilon^{-1} .
$$

To introduce a local time for $Y^{\infty}$, we prove

Proposition 4.1. (a) Let $M_{t}$ be a continuous local martingale, $V_{t}$ be adapted and have continuous sample paths of bounded variation on compacts and $X=M+$ $V$. If $Y(u)=X\left(e^{u}\right) e^{-u / 2}(u \in \mathbf{R})$, then for a.a. $\omega$,

$$
\begin{aligned}
L^{y}(Y)(s, t]= & \lim _{\varepsilon \rightarrow 0+} \int_{s}^{t} I\left(Y_{u} \in[y, y+\varepsilon]\right) e^{-u} d\left(\langle M\rangle\left(e^{u}\right)\right) \varepsilon^{-1}, \\
& -\infty<s<t<\infty, y \in \mathbf{R},
\end{aligned}
$$

defines a family of random measures on the line, which we call the local time of $Y$. $L^{y}(Y)(s, t]$ is jointly right-continuous in $y$ and continuous in $(s, t)$ a.s. Moreover,

$$
L_{t}^{0}(X)=\int_{-\infty}^{\log t} e^{u / 2} d L_{u}^{0}(Y) \quad \forall t \geq 0 \text { a.s. }
$$

(b) If, in addition, $Y(u)$ and $Y(-u)$ are equal in law, then $t \rightarrow L_{t}^{0}(X)$ is equal in law to

$$
t \rightarrow \int_{\log 1 / t}^{\infty} e^{-u / 2} d L_{u}^{0}(Y) .
$$

PROOF. Since $Y_{n}(u)=\{Y(u) \mid u \geq-n\}$ is a continuous semimartingale (integration by parts), the existence of

$$
\begin{aligned}
L_{t}^{y}\left(Y_{n}\right)=\lim _{\varepsilon \rightarrow 0+} \int_{-n}^{t} I\left(Y_{u} \in[y, y+\varepsilon]\right) e^{-u} d\left(\langle M\rangle\left(e^{u}\right)\right) \varepsilon^{-1}, & \\
& t \geq-n, y \in \mathbf{R}, n \in \mathbf{N} \text { a.s., }
\end{aligned}
$$

proves the existence and continuity properties of $L_{t}^{y}(Y)$. 
For a.a. $\omega$ and $0<t_{0}<t$,

$$
\begin{aligned}
L_{t}^{0}(X)-L_{t_{0}}^{0}(X) & =\lim _{\varepsilon \rightarrow 0+} \int_{t_{0}}^{t} I(\sqrt{s} Y(\log s) \in[0, \varepsilon]) d\langle M\rangle_{s} \varepsilon^{-1} \\
& =\lim _{\varepsilon \rightarrow 0+} \int_{\log t_{0}}^{\log t} I\left(e^{u / 2} Y(u) \in[0, \varepsilon]\right) e^{u} e^{-u} d\left(\langle M\rangle\left(e^{u}\right)\right) \varepsilon^{-1} \\
& =\lim _{\varepsilon \rightarrow 0+} \int_{0}^{\varepsilon t_{0}^{-1 / 2}} \int_{\log t_{0}}^{\log \left(t \wedge \varepsilon^{2} y^{-2}\right)} e^{u} d L_{u}^{y}(Y) d y \varepsilon^{-1}
\end{aligned}
$$

Use an integration by parts and the right-continuity of local time in the space variable to conclude that

$$
\lim _{y \rightarrow 0+} \int_{s_{1}}^{s_{2}} e^{u} d L_{u}^{y}(Y)=\int_{s_{1}}^{s_{2}} e^{u} d L_{u}^{0}(Y)
$$

where the convergence is uniform for $\left(s_{1}, s_{2}\right)$ in compacts. Therefore

$$
\begin{aligned}
L_{t}^{0}(X)-L_{t_{0}}^{0}(X) & =\lim _{\varepsilon \rightarrow 0+} \int_{0}^{\varepsilon t_{0}^{-1 / 2}} \int_{\log t_{0}}^{\log \left(t \wedge \varepsilon^{2} y^{-2}\right)} e^{u} d L_{u}^{0}(Y) d y \varepsilon^{-1} \\
& =\lim _{\varepsilon \rightarrow 0+} \int_{\log t_{0}}^{\log t}\left(\int_{0}^{\varepsilon e^{-u / 2}} d y\right) \varepsilon^{-1} e^{u} d L_{u}^{0}(Y) \\
& =\int_{\log t_{0}}^{\log t} e^{u / 2} d L_{u}^{0}(Y) .
\end{aligned}
$$

Let $t_{0} \downarrow 0$ to obtain (a). (b) is obvious.

Note that $(X, Y)=\left(X^{\infty}, Y^{\infty}\right)$ satisfy the hypotheses of $(\mathrm{a})$ and $(\mathrm{b})$.

The following standard results on stochastic differential equations with reflecting boundary conditions will prove useful.

Proposition 4.2. Let $W_{t}$ be a 1-dimensional Brownian motion.

(a) If $b$ is Lipschitz continous on $[0, \infty)$, then there is a unique solution (both pathwise unique and unique in law) of

$$
X_{t}=W_{t}+\int_{0}^{t} b\left(X_{s}\right) d s+\frac{1}{2} L_{t}^{0}(X), \quad X_{t} \geq 0 .
$$

(b) If $a>0$ and $b$ is Lipschitz continuous on $[0, a]$, then there is a unique solution (both pathwise and in law) of

$$
X_{t}=W_{t}+\int_{0}^{t} b\left(X_{s}\right) d s+\frac{1}{2} L_{t}^{0}(X)-\frac{1}{2} L_{t}^{a}(X), \quad X_{t} \in[0, a] .
$$

Proof. (a) is essentially due to Skorokhod (1961) (see also El Karoui and Chaleyat-Maurel (1978, Proposition I.2.1) for the proof that the above equation is equivalent to that considered by Skorokhod). The method of Yamada and Watanabe (1971) shows that uniqueness in law holds.

(b) follows easily from (a) [see the remark of Skorokhod (1961, p. 265)].

Although $\psi_{0}$ may be explicitly described in terms of confluent hypergeometric functions, the elementary properties we will need can easily be derived from the equation $A \psi_{0}=-\lambda_{0} \psi_{0}, \psi_{0}( \pm c)=0$. 
LEMMA 4.3. (a) $\psi_{0}$ is an even function such that $\psi_{0}^{\prime}<0$ on $(0, c]$ and $\psi_{0}^{\prime \prime}<0$ on $[-c, c]$.

(b) $\psi_{0}^{\prime} / \psi_{0}$ is strictly decreasing on $(-c, c)$.

(c) If $0<\tilde{c}<c$, then

$$
\psi_{0}^{\prime}(c)(x) / \psi_{0}(c)(x)>\psi_{0}^{\prime}(\tilde{c})(x) / \psi_{0}(\tilde{c})(x) \quad \forall x \in(0, \tilde{c}) .
$$

PROOF. (a) Since $\psi_{0}$ is the unique eigenfunction for $-\lambda_{0}, \psi_{0}$ must be even, and hence $\psi_{0}^{\prime}(0)=0$. If $\psi_{0}^{\prime}(x) \leq 0$ for some $x$ in $[0, c)$, then $\psi_{0}^{\prime \prime}(x) \leq-2 \lambda_{0} \psi_{0}(x)<0$. Taking $x=0$, we see that $x_{0} \equiv \inf \left\{x>0 \mid \psi_{0}^{\prime}(x) \geq 0\right\}>0(\inf \varnothing=\infty)$. If $x_{0}<\infty$, then

$$
\psi_{0}^{\prime}\left(x_{0}\right)=\int_{0}^{x_{0}} \psi_{0}^{\prime \prime}(x) d x<0,
$$

a contradiction. Therefore $\psi_{0}^{\prime}(x)<0$ on $(0, c]$ and so is

$$
\psi_{0}^{\prime \prime}(x)=x \psi_{0}^{\prime}(x)-2 \lambda_{0} \psi_{0}(x) .
$$

By symmetry $\psi_{0}^{\prime \prime}<0$ on $[-c, c]$.

(b) $\left(\psi_{0}^{\prime} / \psi_{0}\right)^{\prime}=\left(\psi_{0}^{\prime \prime} \psi_{0}-\psi_{0}^{\prime 2}\right) \psi_{0}^{-2}<0$ on $(-c, c)$.

(c) Let

$$
f_{c}(x)=\psi_{0}^{\prime}(c)(x) / \psi_{0}(c)(x)=\frac{d}{d x} \log \left(\psi_{0}(c)(x)\right) \text { for }|x|<c .
$$

Then $A \psi_{0}=-\lambda_{0} \psi_{0}$ leads to

$$
f_{c}^{\prime}(x)+f_{c}(x)^{2}-(x / 2) f_{c}(x)=-2 \lambda_{0}(c), \quad|x|<c .
$$

Therefore if $0<\tilde{c}<c$,

$$
f_{c}^{\prime}(0)=-2 \lambda_{0}(c)>-2 \lambda_{0}(\tilde{c})=f_{\tilde{c}}^{\prime}(0),
$$

where we have used the strict monotonicity of $\lambda_{0}$ [Greenwood and Perkins (1983, Proposition 2)]. Let

$$
x_{1}=\inf \left\{x \in(0, \tilde{c}) \mid f_{c}^{\prime}(x)=f_{\tilde{c}}^{\prime}(x)\right\}>0 \quad \text { (by the above), }
$$

where inf $\varnothing=\infty$. If $x_{1}<\infty$, then

$$
f_{c}\left(x_{1}\right)=\int_{0}^{x_{1}} f_{c}^{\prime}(y) d y>\int_{0}^{x_{1}} f_{\tilde{c}}^{\prime}(y) d y=f_{\tilde{c}}\left(x_{1}\right)
$$

and, since $f_{c}(x)<0$ on $(0, c)$ (by (b) and $\left.f_{c}(0)=0\right)$, we get

$$
f_{c}\left(x_{1}\right)^{2}<f_{\tilde{c}}\left(x_{1}\right)^{2}, \quad-x_{1} f_{c}\left(x_{1}\right) / 2<-x_{1} f_{\tilde{c}}\left(x_{1}\right) / 2 .
$$

On the other hand, (4.2) implies (if $x_{1}<\infty$ )

$$
\left(f_{c}\left(x_{1}\right)^{2}-\frac{x_{1}}{2} f_{c}\left(x_{1}\right)\right)-\left(f_{\tilde{c}}\left(x_{1}\right)^{2}-\frac{x_{1}}{2} f_{\tilde{c}}\left(x_{1}\right)\right)=2 \lambda_{0}(\tilde{c})-2 \lambda_{0}(c)>0,
$$

which contradicts (4.3). Therefore $x_{1}=\infty$, and hence $f_{c}(x)>f_{\tilde{c}}(x)$ for $x \in$ $(0, \tilde{c})$.

We are indebted to Terry Lyons for the proof of the following key result. 
LEMMA 4.4. There is a constant $K$ such that for all $x>K$ and $t>0$,

$$
P\left(\int_{0}^{T_{Y \infty}(0)+t} e^{-\left(s-T_{Y \infty}(0)\right) / 2} d L_{s}^{0}\left(Y^{\infty}\right) \geq x\right) \leq \exp \left\{\frac{-(x-K)^{2}}{2}\right\} .
$$

PROOF. If $Y(t)=Y^{\infty}\left(T_{Y^{\infty}}(0)+t\right)$ and $g$ is as in (2.22), then that result, with $s=T_{Y \infty}(0)$, shows there is an $\left\{\mathcal{F}_{t}^{Y}\right\}$-Brownian motion, $W_{0}$, such that

$$
Y(t)=W_{0}(t)+\int_{0}^{t} g\left(Y_{s}\right) d s
$$

An application of Tanaka's formula shows there is an $\left\{\mathcal{F}_{t}^{Y}\right\}$-Brownian motion, $W$, such that

$$
\begin{aligned}
|Y(t)| & =W(t)+\int_{0}^{t} \operatorname{sgn}\left(Y_{s}\right) g\left(Y_{S}\right) d s+L_{t}^{0}(Y) \\
& =W(t)+\int_{0}^{t} g\left(\left|Y_{s}\right|\right) d s+L_{t}^{0}(Y)
\end{aligned}
$$

where we have the fact that $\psi_{0}$ is an even function. By Proposition 4.2(b) there is a unique solution $\hat{Y}(t)$ of

$$
\hat{Y}(t)=W(t)+\int_{0}^{t} g\left(\hat{Y}_{s}\right) d s+\frac{1}{2} L_{t}^{0}(\hat{Y})-\frac{1}{2} L_{t}^{c / 2}(\hat{Y}), \quad \hat{Y}_{t} \in\left[0, \frac{c}{2}\right] .
$$

If $V(t)=|Y(t)|-\hat{Y}(t)$, and $k$ is chosen so that $|g(x)-g(y)| \leq k|x-y|$ for $x, y \in$ $[0, c / 2]$, then

$$
\begin{aligned}
V(t)^{-}= & -\int_{0}^{t} I\left(V_{s}<0\right) d V_{s} \\
= & \int_{0}^{t} I\left(\left|Y_{s}\right|<\hat{Y}_{s}\right)\left(g\left(\hat{Y}_{s}\right)-g\left(\left|Y_{s}\right|\right)\right) d s-\int_{0}^{t} I\left(0<\hat{Y}_{s}\right) d L_{s}^{0}(Y) \\
& +\frac{1}{2} \int_{0}^{t} I\left(\left|Y_{s}\right|<0\right) d L_{s}^{0}(\hat{Y})-\frac{1}{2} \int_{0}^{t} I\left(|Y(s)|<\frac{c}{2}\right) d L_{s}^{c / 2}(\hat{Y}) \\
\leq & k \int_{0}^{t}\left(\left|Y_{s}\right|-\hat{Y}_{s}\right)^{-} d s=k \int_{0}^{t} V_{s}^{-} d s .
\end{aligned}
$$

Take expected values in the above and apply Gronwall's Lemma to see that $V_{t}^{-}=0$ a.s., and hence $\hat{Y}_{t} \leq\left|Y_{t}\right| \forall t \geq 0$ a.s. Therefore for a.a. $\omega$ and all $0 \leq t_{1}<t_{2}$, we have

$$
\begin{aligned}
L_{t_{2}}^{0}(Y)-L_{t_{1}}^{0}(Y) & =\lim _{\varepsilon \rightarrow 0+} \frac{1}{2} \int_{t_{1}}^{t_{2}} I\left(\left|Y_{s}\right| \in[0, \varepsilon]\right) d s \varepsilon^{-1} \\
& \leq \lim _{\varepsilon \rightarrow 0+} \frac{1}{2} \int_{t_{1}}^{t_{2}} I\left(\hat{Y}_{s} \in[0, \varepsilon]\right) d s \varepsilon^{-1} \\
& =\frac{1}{2}\left(L_{t_{2}}^{0}(\hat{Y})-L_{t_{1}}^{0}(\hat{Y})\right)
\end{aligned}
$$

and hence

$$
\int_{0}^{t} e^{-s / 2} d L_{s}^{0}(Y) \leq \frac{1}{2} \int_{0}^{t} e^{-s / 2} d L_{s}^{0}(\hat{Y}) \quad \forall t \geq 0 \text { a.s. }
$$


Now let $f(x)=(2 / c)(x-c / 4)^{2}$ and use Itô's formula to see that

$$
\begin{aligned}
& \frac{1}{2} L_{t}^{0}(\hat{Y})+\frac{1}{2} L_{t}^{c / 2}(\hat{Y})=f(0)-f\left(\hat{Y}_{t}\right)+\int_{0}^{t} f^{\prime}\left(\hat{Y}_{s}\right) d W_{s}+\int_{0}^{t} f^{\prime} g\left(\hat{Y}_{s}\right) d s+\frac{2}{c} t \\
& \Rightarrow \frac{1}{2} \int_{0}^{t} e^{-s / 2} d L_{s}^{0}(\hat{Y}) \leq \int_{0}^{t} e^{-s / 2} d\left(f(0)-f\left(\hat{Y}_{s}\right)\right) \\
& \left.\quad+K_{1} \int_{0}^{t} e^{-s / 2} d s+\int_{0}^{t} e^{-s / 2} f^{\prime}\left(\hat{Y}_{s}\right) d W_{s} \quad \text { (for some } K_{1}>0\right) .
\end{aligned}
$$

An integration by parts shows that the first term is bounded, and, by writing the stochastic integral as a time change of Brownian motion $(\tilde{W})$, we may bound the right side by

$$
K_{2}+\tilde{W}\left(\int_{0}^{t} e^{-s} f^{\prime}\left(\hat{Y}_{s}\right)^{2} d s\right) \leq K_{2}+\sup _{u \leq 1-e^{-t}} \tilde{W}(u),
$$

where $K_{2}<\infty$ is independent of $t$. We have used the facts that on [0,c/2], $f$ and $g$ are bounded and $\left|f^{\prime}\right|<1$. Combine the above with (4.5) to get

$$
\int_{0}^{t} e^{-s / 2} d L_{s}^{0}(Y) \leq K_{2}+\sup _{u \leq 1} \tilde{W}(u)
$$

and, hence, the required result.

THEOREM 4.5.

$$
\limsup _{t \rightarrow 0+} L_{t}^{0}\left(X^{\infty}\right)\left(2 t \log \log \frac{1}{t}\right)^{-1 / 2}=1 \quad \text { a.s. }
$$

ProOF. Fix $\varepsilon>0$. By Lemma 4.4 there is a universal constant $N_{0}$ such that if $N \geq N_{0}$ and $n \in \mathbf{N}$,

$$
\begin{aligned}
& P\left(\int_{n N}^{(n+1) N} e^{-(s-n N) / 2} d L_{s}^{0}\left(Y^{\infty}\right)>(1+\varepsilon)(2 \log n N)^{1 / 2}\right) \\
& \quad=P\left(\int_{0}^{N} e^{-s / 2} d L_{s}^{0}\left(Y^{\infty}\right)>(1+\varepsilon)(2 \log n N)^{1 / 2}\right) \quad\left(Y^{\infty} \text { is stationary }\right) \\
& \quad \leq(n N)^{-(1+\varepsilon / 2)^{2}} .
\end{aligned}
$$

Choose $N \geq N_{0}$ and let

$$
S(u)=\int_{u}^{\infty} e^{-(s-u) / 2} d L_{s}^{0}\left(Y^{\infty}\right)
$$

The Borel-Cantelli Lemma implies that for a.a. $\omega$ and large enough $k$,

$$
\begin{aligned}
S(k N)(2 \log k N)^{-1 / 2} \leq & \sum_{n=k}^{\infty} \int_{n N}^{(n+1) N} e^{-(s-n N) / 2} d L_{s}^{0}\left(Y^{\infty}\right) \\
& \times \exp \{-(n-k) N / 2\}(2 \log k N)^{-1 / 2} \\
\leq & (1+\varepsilon) \sum_{n=k}^{\infty} \exp \left\{\frac{-(n-k) N}{2}\right\}\left(\frac{\log n N}{\log k N}\right)^{1 / 2} \\
\leq & \left(1+\varepsilon+\delta_{N}\right),
\end{aligned}
$$


where $\lim _{N \rightarrow \infty} \delta_{N}=0$. By the stationarity of $S$ there is a $k_{0}(\omega)<\infty$ a.s. such that for all $k \geq k_{0}(\omega), k \in \mathbf{N}$,

$$
S(y+k N)(2 \log k N)^{-1 / 2} \leq 1+\varepsilon+\delta_{N} \quad \text { for all } y \in L_{N}=\left\{i / N \mid i=0,1, \ldots, N^{2}\right\} .
$$

If $u \geq k_{0} N$, and $k \geq k_{0}, k \in \mathbf{N}$ and $y \in L_{N}$ are chosen so that $u \in[k N+y, k N+$ $\left.y+N^{-1}\right)$, then

$$
S(u)(2 \log u)^{-1 / 2} \leq S(y+k N) e^{(2 N)^{-1}}(2 \log k N)^{-1 / 2} \leq\left(1+\varepsilon+\delta_{N}\right) e^{(2 N)^{-1}} .
$$

Let $N \rightarrow \infty$ and $\varepsilon \downarrow 0$ to see that

$$
\limsup _{u \rightarrow \infty} S(u)(2 \log u)^{-1 / 2} \leq 1 \quad \text { a.s. }
$$

Proposition 4.1(b) shows that the processes $L_{t}^{0}\left(X^{\infty}\right) t^{-1 / 2}$ and $S(\log 1 / t)(t>0)$ are equal in law, whence

$$
\limsup _{t \rightarrow 0+} L_{t}^{0}\left(X^{\infty}\right)\left(2 t \log \log \frac{1}{t}\right)^{-1 / 2} \leq 1 \text { a.s. }
$$

To obtain the converse inequality, the above equivalence shows that it suffices to prove

$$
\limsup _{u \rightarrow \infty} \int_{u}^{\infty} e^{-(s-u) / 2} d L_{s}^{0}(Y)(2 \log u)^{-1 / 2} \geq 1 \quad \text { a.s. }
$$

where $Y(t)=Y^{\infty}\left(T_{Y^{\infty}}(0)+t\right)$, as in Lemma 4.4. If $W$ is the Brownian motion in (4.4), Proposition 4.2(a) shows there is a unique solution $Y^{1}$ of

$$
Y_{t}^{1}=W_{t}-\frac{1}{2} \int_{0}^{t} Y_{s}^{1} d s+\frac{1}{2} L_{t}^{0}\left(Y^{1}\right), \quad Y^{1} \geq 0
$$

Moreover, if $Z_{t}=Z_{0}\left(t+T_{Z_{0}}(0)\right)$, where $Z_{0}(w)=\tilde{B}\left(e^{w}\right) e^{-w / 2}$ is the stationary Ornstein-Uhlenbeck process with generator $A$, then Tanaka's formula and the uniqueness in law for (4.7) show that $Y^{1}$ has the same law as $|Z|$. If $V=Y^{1}-|Y|$, then (4.4), (4.7) and Tanaka's formula show that

$$
\begin{aligned}
V_{t}^{-}= & \int_{0}^{t} I\left(Y_{s}^{1}<\left|Y_{s}\right|\right)\left(\frac{1}{2} Y_{s}^{1}-\frac{1}{2}\left|Y_{s}\right|+\frac{\psi_{0}^{\prime}}{\psi_{0}}\left(\left|Y_{s}\right|\right)\right) d s \\
& -\frac{1}{2} \int_{0}^{t} I\left(0<\left|Y_{s}\right|\right) d L_{s}^{0}\left(Y^{1}\right)+\int_{0}^{t} I\left(Y_{s}^{1}<0\right) d L_{s}^{0}(Y) \leq 0 .
\end{aligned}
$$

We have used the fact (Lemma 4.3(b)) that $\psi_{0}^{\prime} / \psi_{0} \leq 0$ on $[0, c]$. Therefore $Y_{t}^{1} \geq\left|Y_{t}\right|$ for $t \geq 0$ a.s. and, as in the proof of Lemma 4.4, we may conclude that

$$
\int_{u}^{\infty} e^{-(s-u) / 2} d L_{s}^{0}(Y) \geq \frac{1}{2} \int_{u}^{\infty} e^{-(s-u) / 2} d L_{s}^{0}\left(Y^{1}\right) \quad \text { for all } u \geq 0 \text { a.s. }
$$

The process on the right side of (4.8) is equal in law to

$$
e^{(T+u) / 2} \int_{-\infty}^{-u-T} e^{t / 2} d L_{t}^{0}\left(Z_{0}\right)=e^{(T+u) / 2} L_{e^{-(u+T)}}^{0}(\tilde{B}),
$$


where $-T=\sup \left\{t \leq 0 \mid Z_{0}(t)=0\right\}$, and we have used Proposition 4.1 with $(X, Y)=$ $\left(\tilde{B}, Z_{0}\right)$ to obtain the above inequality. The law of the iterated logarithm for local time shows that

$$
\limsup _{u \rightarrow \infty} e^{(T+u) / 2} L_{e^{-(u+T)}}^{0}(\tilde{B})(2 \log u)^{-1 / 2}=1 \quad \text { a.s. }
$$

Therefore the above together with (4.8) implies (4.6) and thus completes the proof.

Although conditioning $B_{t}$ to lie within square root boundaries does not affect the "lim sup behaviour" of its local time, we have already indicated in the introduction why it is reasonable to expect that this conditioning will alter its "lim inf behaviour". Further evidence of this fact is obtained by noting that

$$
t=\int_{-c \sqrt{t}}^{c \sqrt{t}} L_{t}^{x}\left(X^{\infty}\right) d x \leq 2 c \sqrt{t} \sup _{x} L_{t}^{x}\left(X^{\infty}\right)
$$

and therefore

$$
\sup _{x} L_{t}^{x}\left(X^{\infty}\right) \geq(2 c)^{-1} \sqrt{t} \text { for all } t \geq 0 .
$$

This should be compared with the following result of Kesten (1965):

$$
\liminf _{t \rightarrow 0+} \sup _{x} L_{t}^{x}(B) t^{-1 / 2}\left(\log \log \frac{1}{t}\right)^{1 / 2}=\gamma \in(0, \infty) .
$$

Recall the diffusion process $\tilde{Y}$ and its associated measures $\left\{\tilde{P}^{y}\right\}$ that were introduced after Theorem 2.5.

LEMMA 4.6.

$$
\begin{aligned}
\lim _{t \rightarrow \infty} \sup _{0 \leq x \leq c} \mid \tilde{P}^{x}\left(T_{\tilde{Y}}(0)>t\right) e^{\left(\lambda_{0}(0, c)-\lambda_{0}(-c, c)\right) t} \psi_{0}(-c, c)(x) \\
\quad-\psi_{0}(0, c)(x) \int_{0}^{c} \psi_{0}(-c, c)(y) \psi_{0}(0, c)(y) m(d y) \mid=0 .
\end{aligned}
$$

ProOF. (2.19) implies that for $x \in(0, c)$,

$$
\begin{aligned}
\tilde{P}^{x}\left(T_{\tilde{Y}}(0)>t\right)= & E^{x}\left(I\left(T_{\tilde{Z}}(0)>t, \rho(-c, c)>t\right) \psi_{0}(-c, c)\left(\tilde{Z}_{t}\right)\right) \\
& \times \psi_{0}(-c, c)(x)^{-1} e^{\lambda_{0}(-c, c) t} \\
= & e^{\left(\lambda_{0}(-c, c)-\lambda_{0}(0, c)\right) t}\left[e^{\lambda_{0}(0, c) t} E^{x}\left(I(\rho(0, c)>t) \psi_{0}(-c, c)\left(\tilde{Z}_{t}\right)\right)\right] \\
& \times \psi_{0}(-c, c)(x)^{-1}
\end{aligned}
$$

Now use the eigenfunction expansion (2.14) and argue as in the proof of Theorem 1.1 in Uchiyama (1980) to get

$$
\begin{aligned}
E^{x}(I(\rho(0, c)> & \left.t) \psi_{0}(-c, c)\left(\tilde{Z}_{t}\right)\right) \\
& =e^{-\lambda_{0}(0, c) t}\left[\psi_{0}(0, c)(x) \int_{0}^{c} \psi_{0}(-c, c)(y) \psi_{0}(0, c)(y) m(d y)+\tilde{r}(t, x)\right],
\end{aligned}
$$

where for some $K>0$,

$$
|\tilde{r}(t, x)| \leq K e^{-\left(\lambda_{1}(0, c)-\lambda_{0}(0, c)\right) t} \quad \text { for all } x \in[0, c], t \geq 0 .
$$

The proof is completed by substituting the above result into (4.9).

Recall that $\alpha(c)=\left[2\left(\lambda_{0}(0, c)-\lambda_{0}(-c, c)\right)\right]^{-1}$. 
THEOREM 4.7 .

$$
\liminf _{t \rightarrow 0+} L_{t}^{0}\left(X^{\infty}\right) t^{-1 / 2}\left(\log \frac{1}{t}\right)^{\theta}=\left\{\begin{array}{l}
\infty \quad \text { if } \theta>\alpha(c), \\
0 \quad \text { if } \theta \leq \alpha(c),
\end{array}\right.
$$

Proof. Fix $\theta>\alpha(c)$. To prove the above lim inf is infinite, it suffices to show (by Proposition 4.1(b) and an elementary interpolation argument) that

$$
\liminf _{n \rightarrow \infty} \int_{n}^{\infty} e^{-(s-n) / 2} d L_{s}^{0}\left(Y^{\infty}\right) n^{\theta} \geq 1 \quad \text { a.s. }(n \in \mathbf{N})
$$

If

$$
\sigma_{n}=\inf \left\{t \geq n \mid L_{t}^{0}\left(Y^{\infty}\right)-L_{n}^{0}\left(Y^{\infty}\right) \geq 1\right\}
$$

then

$$
P\left(\int_{n}^{\infty} e^{-(s-n) / 2} d L_{s}^{0}\left(Y^{\infty}\right) n^{\theta}<1\right) \leq P\left(e^{-\left(\sigma_{n}-n\right) / 2}<n^{-\theta}\right)=P\left(\sigma_{0}>2 \theta \log n\right),
$$

where we have used the stationarity of $Y^{\infty}$ in the last equality. Let $Y(t)=$ $Y^{\infty}\left(T_{Y^{\infty}}(0)+t\right)$, and let $T_{L}(t)$ denote the right-continuous inverse of $L_{t}^{0}(Y)$. Therefore by the strong Markov property we have

$$
\sigma_{0}=T_{Y^{\infty}}(0)+T_{L}(1), \quad T_{Y^{\infty}}(0) \text { and } T_{L}(1) \text { are independent. }
$$

$T_{L}(t)$ is a subordinator and therefore

$$
E\left(e^{-\lambda T_{L}(t)}\right)=\exp \left\{-t\left(b \lambda+\int_{0}^{\infty}\left(1-e^{-1 \lambda x}\right) \nu(d x)\right)\right\}
$$

where $\nu$ is the Lévy measure of $T_{L}$ and $b \geq 0$. (4.13) is usually stated for $\lambda>0$ but in fact holds for $\lambda \in\left(\lambda^{\prime}, \infty\right)$, where

$$
\lambda^{\prime}=\inf \left\{\lambda \mid \int_{0}^{\infty} e^{-\lambda x} \nu(d x)<\infty\right\}
$$

(see Itô (1970, Theorem 4.5) and note that $\alpha$ may be negative in his arguments). If $V=\inf \left\{t \mid \Delta T_{L}(t) \geq 1\right\}$ and $\gamma(d x)$ is the law of $Y\left(T_{L}(V-)+1\right)$, then for $t \geq 2$,

$$
\begin{aligned}
\nu[t, \infty) & =\nu[1, \infty) P\left(\Delta T_{L}(V) \geq t\right) \quad(\text { Itô }(1970, \text { Theorem 4.4A })) \\
& =\nu[1, \infty) \int_{-c}^{c} \tilde{P}^{x}\left(T_{\tilde{Y}}(0) \geq t-1\right) \gamma(d x) \\
& \leq \nu[1, \infty) \int_{-c}^{c} \tilde{E}^{x}\left(\tilde{P}^{\tilde{Y}(1)}\left(T_{\tilde{Y}}(0) \geq t-2\right)\right) \gamma(d x) .
\end{aligned}
$$

$$
\begin{aligned}
& \therefore e^{\left(\lambda_{0}(0, c)-\lambda_{0}(-c, c)\right) t} \nu[t, \infty) \\
& \leq \nu[1, \infty) \int_{-c}^{c} \int_{-c}^{c} \hat{p}^{c}(1, x, y) \psi_{0}(-c, c)(x)^{-1} e^{\lambda_{0}(-c, c)} e^{\left(\lambda_{0}(0, c)-\lambda_{0}(-c, c)\right) t} \\
& \quad \times \tilde{P}^{y}\left(T_{\tilde{Y}}(0) \geq t-2\right) \psi_{0}(-c, c)(y) m(d y) \gamma(d x) .
\end{aligned}
$$

(2.16) and Lemma 4.6 show that the right side of (4.14) remains bounded as $t \rightarrow \infty$, and therefore by (4.13),

$$
E\left(e^{\lambda T_{L}(1)}\right)<\infty \quad \text { if } \lambda<\lambda_{0}(0, c)-\lambda_{0}(-c, c)
$$


The required asymptotics for $P\left(T_{Y^{\infty}}(0)>t\right)$ are easily obtained from Lemma 4.6 as follows:

$$
\begin{aligned}
& e^{\left(\lambda_{0}(0, c)-\lambda_{0}(-c, c)\right) t} P\left(T_{Y \infty}(0)>t\right) \\
& \quad=\int_{-c}^{c} e^{\left(\lambda_{0}(0, c)-\lambda_{0}(-c, c)\right) t} \tilde{P}^{x}\left(T_{\tilde{Y}}(0)>t\right) \psi_{0}(-c, c)(x)^{2} m(d x) . \\
& \quad \sim 2\left(\int_{0}^{c} \psi_{0}(0, c)(x) \psi_{0}(-c, c)(x) m(d x)\right)^{2} \quad \text { as } t \rightarrow \infty .
\end{aligned}
$$

This together with (4.12) and (4.15) shows that

$$
E\left(e^{\lambda \sigma_{0}}\right)<\infty \text { for } \lambda<\lambda_{0}(0, c)-\lambda_{0}(-c, c)=(2 \alpha(c))^{-1} .
$$

If $\theta^{\prime} \in\left(\theta^{-1}, \alpha(c)^{-1}\right)$, use (4.11) and the above to conclude that

$$
P\left(\int_{n}^{\infty} e^{-(s-n) / 2} d L_{s}^{0}\left(Y^{\infty}\right) n^{\theta}<1\right) \leq E\left(e^{\left(\theta^{\prime} / 2\right) \sigma_{0}}\right) n^{-\theta^{\prime} \theta} .
$$

The Borel-Cantelli Lemma now implies (4.10) and thus completes the proof if $\theta>$ $\alpha(c)$.

To complete the proof, we must show that

$$
\liminf _{u \rightarrow \infty} \int_{u}^{\infty} e^{-(s-u) / 2} d L_{s}^{0}\left(Y^{\infty}\right) u^{\alpha(c)}=0 .
$$

Inductively define $\left\{\mathcal{F}_{t}^{Y^{\infty}}\right\}$-stopping times by

$$
\begin{aligned}
V_{0} & =\inf \left\{t \geq 0 \mid Y^{\infty}(t)=0\right\}, \\
U_{i+1} & =\inf \left\{t \geq V_{i}|| Y^{\infty}(t) \mid=c / 2\right\}, \\
V_{i+1} & =\inf \left\{t \geq U_{i+1} \mid Y^{\infty}(t)=0\right\}
\end{aligned}
$$

Lemma 4.6 shows that

$$
P\left(V_{i}-U_{i}>t\right) \sim K e^{-\left(\lambda_{0}(0, c)-\lambda_{0}(-c, c)\right) t} \quad \text { as } t \rightarrow \infty(K \in(0, \infty)),
$$

and the same argument gives

$$
P\left(U_{i+1}-V_{i}>t\right) \sim K^{\prime} e^{-\left(\lambda_{0}(-c / 2, c / 2)-\lambda_{0}(-c, c)\right) t} \quad \text { as } t \rightarrow \infty\left(K^{\prime} \in(0, \infty)\right) .
$$

The strong Markov property shows that $\left\{V_{i}-V_{i-1} \mid i=1,2, \ldots\right\}$ are i.i.d. random variables, which have a finite mean, $\mu$, by the above. (4.16) would follow from

$$
\liminf _{i \rightarrow \infty} \int_{U_{i}}^{\infty} e^{-\left(s-U_{i}\right) / 2} d L_{s}^{0}\left(Y^{\infty}\right) U_{i}^{\alpha(c)}=0 \quad \text { a.s. }
$$

or, equivalently,

$$
\liminf _{i \rightarrow \infty} W_{i} e^{-\left(V_{i}-U_{i}\right) / 2} U_{i}^{\alpha(c)}=0 \quad \text { a.s., }
$$

where

$$
W_{i}=\int_{V_{i}}^{\infty} e^{-\left(s-V_{i}\right) / 2} d L_{s}^{0}\left(Y^{\infty}\right) .
$$

The law of large numbers implies $U_{i} \leq 2 \mu i$ for large $i$ a.s., and so (4.18) will hold if, for every $\varepsilon>0$,

$$
W_{i} e^{-\left(V_{i}-U_{i}\right) / 2}<\varepsilon i^{-\alpha(c)} \quad \text { infinitely often a.s. }
$$


If $\varepsilon>0$ is fixed, then

$$
\begin{gathered}
P\left(e^{-\left(V_{i}-U_{i}\right) / 2}<\varepsilon^{2} i^{-\alpha(c)}\right)=P\left(V_{i}-U_{i}>2 \log \left(\varepsilon^{-2}\right)+2 \alpha(c) \log i\right) \\
\sim K \varepsilon^{-4\left(\lambda_{0}(0, c)-\lambda_{0}(-c, c)\right)} i^{-1} \quad \text { as } i \rightarrow \infty(\text { by }(4.17)) .
\end{gathered}
$$

The Borel-Cantelli Lemma shows that

$$
e^{-\left(V_{i}-U_{i}\right) / 2}<\varepsilon^{2} i^{-\alpha(c)} \quad \text { infinitely often a.s. }
$$

If $\left\{i_{n}(\omega) \mid n \in \mathbf{N}\right\}$ denote the successive times at which the above inequality holds, then $\left\{i_{n}=k\right\} \in \mathcal{F}_{V_{k}}^{Y^{\infty}}$ and so is independent of $W_{k}$ by the strong Markov property. Since the $\left\{W_{k}\right\}$ are identically distributed, we get

$$
P\left(W_{i_{n}}<\varepsilon^{-1}\right)=\sum_{k=n}^{\infty} P\left(i_{n}=k\right) P\left(W_{k}<\varepsilon^{-1}\right)=P\left(W_{1}<\varepsilon^{-1}\right),
$$

and therefore

$$
\begin{aligned}
& P\left(W_{i} e^{-\left(V_{i}-U_{i}\right) / 2}<\varepsilon i^{-\alpha(c)} \text { i.o. }\right) \geq P\left(W_{i_{n}}<\varepsilon^{-1} \text { i.o. }\right) \\
& \quad=\lim _{k \rightarrow \infty} P\left(W_{i_{n}}<\varepsilon^{-1} \text { for some } n \geq k\right) \geq P\left(W_{1}<\varepsilon^{-1}\right) .
\end{aligned}
$$

Note that the extreme right side approaches 1 as $\varepsilon \rightarrow 0+$, while the extreme left side decreases as $\varepsilon \rightarrow 0+$. (4.19) is immediate, and the proof is complete.

It is now an easy matter to derive Theorem 1.2(b) from Theorems 4.5 and 4.7.

PROOF OF THEOREM 1.2. Consider (b) first. Recall the definition of $X^{r, v}$ made after Theorem 3.3. Since $P_{X^{r, v}}$ and $P_{X^{\infty}}$ are equivalent laws on $C[0, v]$ for any $v>0, r \geq 0$ (Theorem 2.3(b) and Remark 3.4), Theorems 4.5 and 4.7 imply for a.a. $\omega$ and all rational $v>0, r \geq 0$ that

$$
\begin{aligned}
& \limsup _{\delta \rightarrow 0+} L_{\delta}^{0}\left(X^{r, v}\right)\left(2 \delta \log \log \frac{1}{\delta}\right)^{-1 / 2}=1, \\
& \liminf _{\delta \rightarrow 0+} L_{\delta}^{0}\left(X^{r, v}\right) \delta^{-1 / 2}\left(\log \frac{1}{\delta}\right)^{\theta}= \begin{cases}\infty & \text { if } \theta>\alpha(c), \\
0 & \text { if } \theta \leq \alpha(c) .\end{cases}
\end{aligned}
$$

Theorem 1.2(b) follows by noting that

$$
\{\tau(t-) \mid \tau(t-)<\tau(t)\}=\left\{S^{r, v} \mid r \geq 0, v>0 \text {, both rational }\right\} \text { a.s. }
$$

The fact that $\lim _{c \rightarrow \infty} \alpha(c)=1$ is immediate from Proposition 2(c) of Greenwood and Perkins (1983), the proof of which we now modify to establish the monotonicity of $\alpha$. Let $\overline{P_{t}^{c}}$ and $\overline{R_{\lambda}^{c}}$ denote the semigroup and resolvent, respectively, of the process, $\overline{Y^{c}}$, obtained by killing $\tilde{Y}^{(c)}$ when it hits 0 (here $\tilde{Y}^{(c)}=\tilde{Y}$ is the process introduced after Theorem 2.5). Use Theorem 2.5 and Lemma 4.3.4 of Knight (1981) (and an easy limiting argument) to conclude that for $\phi \geq 0$ and measurable on $[0, c]$,

$$
\bar{R}_{0}^{(c)} \phi(x)=2 \int_{0}^{c} \phi(y) \tilde{s}^{c}(x \wedge y) d \tilde{m}^{c}(y)=2 \int_{0}^{c} \phi(y) \overline{G_{0}^{c}}(x, y) d m(y),
$$

where

If

$$
\overline{G_{0}^{c}}(x, y)=\int_{0}^{x \wedge y} e^{z^{2} / 2} \psi_{0}(c)(z)^{-2} \psi_{0}(c)(y)^{2} d z
$$

$$
\bar{\psi}_{k}(c)(x)=\psi_{k}(0, c)(x) \psi_{0}(-c, c)(x)^{-1}, \quad x \in[0, c)
$$


then $\left\{\bar{\psi}_{k}(c) \mid k=0,1, \ldots\right\}$ is a complete orthonormal system in $L^{2}\left([0, c], \tilde{m}^{c}\right)$, and for $x \in[0, c)$,

$$
\begin{aligned}
\bar{P}_{t}^{c}\left(\bar{\psi}_{k}(c)\right)(x) & =E^{x}\left(\psi_{k}(0, c)\left(\tilde{Z}_{t}\right) I(\rho(0, c)>t)\right) e^{\lambda_{0}(-c, c) t} \psi_{0}(-c, c)(x)^{-1} \\
& =e^{-\bar{\lambda}_{k}(c) t} \bar{\psi}_{k}(c)(x)
\end{aligned}
$$

Here $\bar{\lambda}_{k}(c)=\lambda_{k}(0, c)-\lambda_{0}(-c, c)>0$, and the last line follows from the eigenfunction expansion of the transition density of the Ornstein-Uhlenbeck process killed when it hits 0 or $c$ (as in Proposition 2.4(a)). Therefore $\overline{R_{0}^{c}} \bar{\psi}_{k}(x)=\bar{\lambda}_{k}(c)^{-1} \bar{\psi}_{k}(x)$ and the norm of $\overline{R_{0}^{c}}$, as an operator on $L^{2}\left([0, c], \tilde{m}^{c}\right)$, is $\left\|\overline{R_{0}^{c}}\right\|=\bar{\lambda}_{0}(c)^{-1}$.

Fix $c>\tilde{c}>0$. If $0 \leq z<y<\tilde{c}$, Lemma 4.3(c) implies

$$
\int_{z}^{y} \psi_{0}^{\prime}(\tilde{c})(u) \psi_{0}(\tilde{c})(u)^{-1} d u<\int_{z}^{y} \psi_{0}^{\prime}(c)(u) \psi_{0}(c)(u)^{-1} d u
$$

and, therefore, by taking exponentials one finds

$$
\psi_{0}(\tilde{c})(y) / \psi_{0}(\tilde{c})(z)<\psi_{0}(c)(y) / \psi_{0}(c)(z), \quad 0 \leq z<y<\tilde{c} .
$$

After extending $\psi_{0}(\tilde{c})$ to $[0, c]$ by setting $\psi_{0}(\tilde{c})(x)=0$ for $x \in(\tilde{c}, c]$, one gets

$$
\begin{aligned}
\bar{\lambda}_{0}(\tilde{c})^{-2}= & \int_{0}^{\tilde{c}}\left(\overline{R_{0}^{\tilde{c}}}\left(\bar{\psi}_{0}(\tilde{c})\right)(x)\right)^{2} \tilde{m}^{\tilde{c}}(d x) \\
= & \int_{0}^{\tilde{c}}\left(\int_{0}^{\tilde{c}} \int_{0}^{x \wedge y} e^{z^{2} / 2} \frac{\psi_{0}(-\tilde{c}, \tilde{c})(y) \psi_{0}(-\tilde{c}, \tilde{c})(x)}{\psi_{0}(-\tilde{c}, \tilde{c})(z)^{2}} d z\right. \\
& \left.\times \psi_{0}(0, \tilde{c})(y) d m(y)\right)^{2} d m(x) \\
& \left.<\int_{0}^{c}\left(\int_{0}^{c} \int_{0}^{x \wedge y} e^{z^{2} / 2} \frac{\psi_{0}(-c, c)(y) \psi_{0}(-c, c)(x)}{\psi_{0}(-c, c)(z)^{2}} d z\right)(y) d m(y)\right)^{2} d m(x) \\
= & \int_{0}^{c}\left(\overline{R_{0}^{c}}\left(\frac{\psi_{0}(0, \tilde{c})}{\psi_{0}(-c, c)}\right)(x)\right)^{2} d \tilde{m}^{c}(x) \\
\leq & \left\|\bar{R}_{0}^{c}\right\|^{2} \int_{0}^{c} \psi_{0}(0, \tilde{c})^{2}(x) d m(x) \\
= & \bar{\lambda}_{0}(c)^{-2} .
\end{aligned}
$$

Therefore $\alpha(c)=\left(2 \bar{\lambda}_{0}(c)\right)^{-1}$ is strictly increasing on $(0, \infty)$.

5. Stochastic integrals and slow points. Throughout this section we work on the filtered probability space $\left(\Omega, \mathcal{F}, \mathcal{F}_{t}, P\right)$ introduced in $\S 1$. Recall also the processes $X^{r, v}$ and filtrations $\left\{\mathcal{H}_{t}^{r, v}\right\}$ defined in $\S 3$. We study the behaviour of a stochastic integral $H \cdot B$ at a slow point in $\{\tau(t-) \mid \tau(t-)<\tau(t)\}$ by first using the results of $\S 3$ to study $H \cdot X^{r, v}(t)$ near $t=0$ and then noting that

$$
\{\tau(t-, \omega) \mid \tau(t-, \omega)<\tau(t, \omega), t \geq 0\}=\left\{S^{r, v} \mid r \geq 0, v>0, \text { both rational }\right\}
$$


THEOREM 5.1. Let $r \geq 0, v>0$ and $H$ be locally bounded and predictable with respect to $\left\{\mathcal{H}_{t}^{r, v}\right\}$. If

$$
\limsup _{\delta \rightarrow 0+}\left|H_{\delta}-H_{0}\right|\left(\log \log \frac{1}{\delta}\right)^{1 / 2}<\infty \quad \text { a.s. }
$$

then

$$
\limsup _{\delta \rightarrow 0+}\left|H \cdot X^{r, v}(\delta)\right| \delta^{-1 / 2}<\infty \quad \text { a.s. }
$$

ProOF. By replacing $H$ with

$$
H_{t}^{(N)}=H_{0}+\left(\left(H_{t}-H_{0}\right) \wedge\left(N(\log \log 1 / t)^{-1 / 2}\right)\right) \vee\left(-N(\log \log 1 / t)^{-1 / 2}\right)
$$

and letting $N \rightarrow \infty$ [use the local character of the stochastic integral; Dellacherie and Meyer (1980, p. 246)], we may assume without loss of generality that for some $N>0$,

$$
\left|H_{t}-H_{0}\right| \leq N(\log \log 1 / t)^{-1 / 2}, \quad t \in\left[0, e^{-1}\right) .
$$

To simplify the notation, take $v=1$ and drop the dependence on $(r, v)$. By Theorem 3.5 there is an $\left\{\mathcal{H}_{t}\right\}$-Brownian motion, $W_{t}$, such that $X=W+V$, where

$$
V_{t}=\int_{0}^{t \wedge 1} \frac{h_{x}}{h}\left(\log \frac{1}{s}, \frac{X_{s}}{\sqrt{s}}\right) \frac{d s}{\sqrt{s}} .
$$

If $|V|_{t}$ denotes the total variation of $V$ on $[0, t]$, Tanaka's formula implies

$$
\begin{aligned}
\left|X_{t}\right| & =\int_{0}^{t} \operatorname{sgn}\left(X_{s}\right) d W_{s}+\int_{0}^{t} \operatorname{sgn}\left(X_{s}\right) \frac{h_{x}}{h}\left(\log \frac{1}{s}, \frac{X_{s}}{\sqrt{s}}\right) \frac{d s}{\sqrt{s}}+L_{t}^{0}(X) \\
& =\hat{W}_{t}-|V|_{t}+L_{t}^{0}(X) .
\end{aligned}
$$

In the last line, $\hat{W}$ is an $\left\{\mathcal{H}_{t}\right\}$-Brownian motion, and we have used Lemma 3.1(b) to show that $\operatorname{sgn}(x) h_{x}(t, x)=-\left|h_{x}(t, x)\right|$. The laws of the iterated logarithm for $\hat{W}$ and $L_{t}^{0}(X)$ (Theorems 4.5, 2.3(b), Remark 3.4) yield

$$
\limsup _{t \rightarrow 0+}|V|_{t}\left(2 t \log \log \frac{1}{t}\right)^{-1 / 2} \leq 2 .
$$

Therefore, for some Brownian motion, $\tilde{W}$, we have for a.a. $\omega$,

$$
\begin{aligned}
& \underset{t \rightarrow 0+}{\limsup }\left|\left(H-H_{0}\right) \cdot X(t)\right| t^{-1 / 2} \\
& \leq \limsup _{t \rightarrow 0+}\left(\left|\left(H-H_{0}\right) \cdot W(t)\right| t^{-1 / 2}+|V|_{t} N\left(t \log \log \frac{1}{t}\right)^{-1 / 2}\right) \\
& \leq \limsup _{t \rightarrow 0+} \sup _{u \leq t N^{2}(\log \log 1 / t)^{-1}}\left|\tilde{W}_{u}\right| t^{-1 / 2}+2 \sqrt{2} N \\
& \leq \limsup _{t \rightarrow 0+} N\left(2 t\left(\log \log \frac{1}{t}\right)^{-1}\right)^{1 / 2}\left(\log \log \left(\frac{1}{t N^{2}}\left(\log \log \frac{1}{t}\right)\right)\right)^{1 / 2} t^{-1 / 2} \\
& \quad+2 \sqrt{2} N \\
& =3 \sqrt{2} N<\infty .
\end{aligned}
$$


Since $\left|H_{0} \cdot X(t)\right| \leq c\left|H_{0}\right| t^{1 / 2}$, this completes the proof.

PROOF OF THEOREM 1.3(a). If $r \geq 0$ and $v>0$, then it is easy to check that $H^{r, v}(t, \omega)=H\left(S^{r, v}(\omega)+t, \omega\right)$ satisfies the hypotheses of the previous theorem, and therefore

$$
\begin{aligned}
& \underset{\delta \rightarrow 0+}{\limsup }\left|H \cdot B\left(S^{r, v}+\delta\right)-H \cdot B\left(S^{r, v}\right)\right| \delta^{-1 / 2} \\
& \quad=\limsup _{\delta \rightarrow 0+}\left|H \cdot X^{r, v}(\delta)\right| \delta^{-1 / 2} \quad \text { (integrals agree for simple } H, \text { etc.) } \\
& \quad<\infty \text { a.s. }
\end{aligned}
$$

Now use (5.1) to finish the proof.

In what follows, we consider a function $\sigma:[0, \infty) \rightarrow[0,1]$ that satisfies the following conditions:

(5.2a) $\sigma$ is strictly increasing near 0 , continuous, and $\sigma(0)=0$.

(5.2b) $\sigma\left(t^{3}\right)$ is concave.

(5.2c) $\lim _{t \rightarrow 0+} \sigma(t)(\log \log 1 / t)^{1 / 2}=\infty$.

Define functions $f$ and $g$ on $\mathbf{R}$ by

$$
f(x)=x^{+} \wedge 1, \quad g(x)=((-x) \vee(-1)) \wedge 1,
$$

and $k$ on $[0, \infty] \times \mathbf{R}$ by

$$
k(s, x)=\left\{\begin{array}{l}
\sigma\left(s^{3}\right) g(x / s) f((c \sqrt{s}-|x|) / s), \quad s>0 \\
0, \quad s=0
\end{array}\right.
$$

Clearly we have

$$
\lim _{s \rightarrow 0+} \sup _{x}|k(s, x)|=0 .
$$

LEMMA 5.2. There is a $K>0$ such that for all $0<s<t \leq s+1$, and $x, y \in \mathbf{R}$, $|k(s, x)-k(t, y)| \leq K\left[\left(|x| s^{-1 / 2}+1\right)(t-s)^{1 / 2}+|x-y|(t-s)^{-1 / 3}+\sigma(t-s)\right]$.

PROOF. If $s, t, x, y$ are as above, then

$$
\begin{aligned}
|k(s, x)-k(t, y)| \leq & \sigma\left(s^{3}\right)\left|f\left(\frac{c \sqrt{s}-|x|}{s}\right)-f\left(\frac{c \sqrt{t}-|y|}{t}\right)\right| \\
& +\sigma\left(s^{3}\right)|g(x / s)-g(y / t)|+\sigma\left((t-s)^{3}\right)
\end{aligned}
$$

where we have used the concavity of $\sigma\left(u^{3}\right)$ to arrive at the last term. If $s<(t-s)^{1 / 3}$, then the right side of (5.4) is clearly bounded by $4 \sigma(t-s)$. Therefore we may assume in what follows that

$$
s \geq(t-s)^{1 / 3}
$$

The right side of (5.4) is then bounded by

$$
\begin{aligned}
c\left|s^{-1 / 2}-t^{-1 / 2}\right|+2|x / s-y / t|+\sigma(t-s) \\
\quad \leq c s^{-3 / 2}(t-s)+2|x|(t-s) / s t+2|x-y| / t+\sigma(t-s) \\
\quad \leq c(t-s)^{1 / 2}+2|x| s^{-1 / 2}(t-s)^{1 / 2}+2|x-y|(t-s)^{-1 / 3}+\sigma(t-s)
\end{aligned}
$$

(by $(5.5))$.

For $r \geq 0, v>0$, let $H^{r, v}(s, \omega)=k\left(s, X^{r, v}(s)\right)$. 
PROPOSITION 5.3. (a) $H^{r, v}$ is bounded and predictable with respect to $\left\{\mathcal{H}_{t}^{r, v}\right\}$ and satisfies $\lim \sup _{\delta \rightarrow 0+}\left|H_{\delta}^{r, v}\right| \sigma(\delta)^{-1}<\infty$ a.s.

(b)

$$
\limsup _{\delta \rightarrow 0+}\left|H^{r, v} \cdot X^{r, v}(\delta)\right|\left(\delta \log \log \frac{1}{\delta}\right)^{-1 / 2} \sigma\left(\frac{\delta^{3}}{2}\right)^{-1} \geq 1 \quad \text { a.s. },
$$

and, in particular,

$$
\limsup _{\delta \rightarrow 0+}\left|H^{r, v} \cdot X^{r, v}(\delta)\right| \delta^{-1 / 2}=\infty \quad \text { a.s. }
$$

Proof. As before, we assume $v=1$ and drop the dependence on $(r, v)$ in the notation. $H^{r, v}$ is bounded and predictable because $X^{r, v}$ is adapted and continuous. Lemma 5.2 shows that if $0<s<t \leq 1$, then

$$
\begin{aligned}
|H(t)-H(s)| & \leq K\left[\left(\left|X_{s}\right| s^{-1 / 2}+1\right)(t-s)^{1 / 2}+\left|X_{t}-X_{s}\right|(t-s)^{-1 / 3}+\sigma(t-s)\right] \\
& \leq K_{1}(\omega)\left[(c+1)(t-s)^{1 / 2}+(t-s)^{1 / 8}+\sigma(t-s)\right] \\
& \leq K_{2}(\omega) \sigma(t-s) \quad(\text { by }(5.2 \mathrm{c})) .
\end{aligned}
$$

The Lévy modulus of continuity for $B$, and therefore $X$, shows that $K_{i}(\omega)<\infty$ a.s. Let $s \downarrow 0$ and use (5.3) to complete the proof of (a).

By Theorem 3.4, there is an $\left\{\mathscr{H}_{t}\right\}$-Brownian motion, $W$, such that

$$
X_{t}=W_{t}+\int_{0}^{t \wedge 1} \frac{h_{x}}{h}\left(\log s^{-1}, \frac{X_{s}}{\sqrt{s}}\right) \frac{d s}{\sqrt{s}} .
$$

If

$$
\phi(t)=\left(2 \int_{0}^{t} \sigma\left(s^{3}\right)^{2} d s \log \log \left(\left(\int_{0}^{t} \sigma\left(s^{3}\right)^{2} d s\right)^{-1} \vee e\right)\right)^{1 / 2}
$$

we claim that

$$
\limsup _{t \rightarrow 0+}\left(\int_{0}^{t} H_{s} d W_{s}\right) \phi(t)^{-1}=1 \text { a.s. }
$$

This would follow easily from

$$
\limsup _{t \rightarrow 0+} \int_{0}^{t}\left(\sigma\left(s^{3}\right) \operatorname{sgn}\left(X_{s}\right)-H_{s}\right)^{2} d s\left(\int_{0}^{t} \sigma\left(s^{3}\right)^{2} d s\right)^{-1}=0 \quad \text { a.s. }
$$

Indeed, the above would imply

$$
\lim _{t \rightarrow 0+}\left|\int_{0}^{t}\left(\sigma\left(s^{3}\right) \operatorname{sgn}\left(X_{s}\right)-H_{s}\right) d W_{s}\right| \phi(t)^{-1}=0 \quad \text { a.s. }
$$

(represent the stochastic integral as a time-changed Brownian motion), and (5.8) would then follow from

$$
\limsup _{t \rightarrow 0+} \int_{0}^{t} \sigma\left(s^{3}\right) \operatorname{sgn}\left(X_{s}\right) d W_{s} \phi(t)^{-1}=1 \quad \text { a.s. }
$$

The definition of $H$ shows that to prove (5.9), it suffices to show that (5.10)

$$
\limsup _{t \rightarrow 0+} \int_{0}^{t} \sigma\left(s^{3}\right)^{2} I\left(\left|X_{s}\right| \leq s \text { or }\left|X_{s}\right| \geq c \sqrt{s}-s\right) d s\left(\int_{0}^{t} \sigma\left(s^{3}\right)^{2} d s\right)^{-1}=0 \text { a.s. }
$$


If $k>0,(2.2)$ shows that

$$
\begin{aligned}
& E\left(\int_{0}^{n^{-k}} \sigma\left(s^{3}\right)^{2} I\left(\left|X_{s}\right| \leq s \text { or }\left|X_{s}\right| \geq c \sqrt{s}-s\right) d s\right)\left(\int_{0}^{(n+1)^{-k}} \sigma\left(s^{3}\right)^{2} d s\right)^{-1} \\
& \leq \int_{0}^{n^{-k}} \sigma\left(s^{3}\right)^{2} \int_{-c}^{c} I(|y| \leq \sqrt{s} \text { or }|y| \geq c-\sqrt{s}) \\
& \quad \times \theta(c)^{-1} s^{-\lambda_{0}} d s\left(\int_{0}^{(n+1)^{-k}} \sigma\left(s^{3}\right)^{2} d s\right)^{-1} \times P^{y}\left(\rho(c)>\log \frac{1}{s}\right) \psi_{0}(c)(y) m(d y) \\
& \leq K_{1} \sigma\left(n^{-3 k}\right)^{2} \sigma\left(\frac{(n+1)^{-3 k}}{2}\right)^{-2} 2(n+1)^{k} \int_{0}^{n^{-k}} s^{1 / 2} s^{\lambda_{0}} s^{-\lambda_{0}} d s \quad \text { (by (2.6)) } \\
& \leq K_{2} n^{-k / 2} .
\end{aligned}
$$

The concavity of $\sigma$ is used in the last line to show that

$$
\sigma\left((n+1)^{-3 k} / 2\right) \geq\left((n /(n+1))^{3 k} / 2\right) \sigma\left(n^{-3 k}\right),
$$

so $K_{2}$ depends only on $k$. Let $k=4$ and apply the Borel-Cantelli Lemma in the above to see that (5.10) holds, and hence so does the claim (5.8). Lemma 3.1(b) shows that

$$
H(s)\left(h_{x} / h\right)\left(\log s^{-1}, X_{s} / \sqrt{s}\right) \geq 0,
$$

and therefore (by (5.7))

$$
\begin{aligned}
\limsup _{t \rightarrow 0+}(H \cdot X)(t) \phi(t)^{-1} & \geq \limsup _{t \rightarrow 0+}(H \cdot W)(t) \phi(t)^{-1} \\
& =1 \quad \text { (by (5.8)). }
\end{aligned}
$$

The first part of (b) is immediate from the obvious inequality

$$
\phi(t) \geq \sigma\left(t^{3} / 2\right)(t \log \log 1 / t)^{1 / 2} \text { for } t \text { small enough, }
$$

and the last part of $(\mathrm{b})$ is clear from $(5.2 \mathrm{c})$.

ProOF OF THEOREM 1.3(b). Let $H(s, \omega)=k\left(A_{s}(\omega), Y_{s}(\omega)\right)$. We will show that $H$ is the required integrand. The jumps of $(A, Y)$ may be covered by the predictable times

$$
\begin{aligned}
T_{r} & =\inf \left\{s>r|| Y_{s-} \mid s^{-1 / 2} \geq c\right\} \\
& =\lim _{n \rightarrow \infty} \inf \left\{s>r|| Y_{s-} \mid s^{-1 / 2} \geq c-\frac{1}{n}\right\}, \quad r \in Q^{\geq 0} .
\end{aligned}
$$

The jumps of $(A, Y)$ at $T_{r}$ are $\mathcal{F}_{T_{r^{-}}}$-measurable because $A_{T_{r}}=Y_{T_{r}}=0$. Therefore $(A, Y)$ and $H$ are both $\left\{\mathcal{F}_{t}\right\}$-predictable.

Assume $0<s<t \leq s+1 \leq T$. If $\tau^{-}(s)=\tau^{-}(t)$, then just as in (5.6) one sees that

$$
|H(s)-H(t)| \leq K_{T}^{1}(\omega) \sigma(t-s), \quad K_{T}^{1}(\omega)<\infty \text { for all } T>0 \text { a.s. }
$$


Assume that $\tau^{-}(s)<\tau^{-}(t)$, which implies $\tau^{+}(s) \leq \tau^{-}(t) \leq t \leq T$. (5.11) implies

$$
\left|H\left(\left(\tau_{s}^{+}\right)-\right)-H(s)\right| \leq K_{T}^{1}(\omega) \sigma\left(\tau_{s}^{+}-s\right)
$$

and

$$
\left|H(t)-H\left(\tau_{t}^{-}\right)\right| \leq K_{T}^{1}(\omega) \sigma\left(t-\tau_{t}^{-}\right) .
$$

Clearly $H\left(\tau_{t}^{-}\right)=0$, and the factor $f((c \sqrt{s}-|x|) / s)$ in the definition of $k$ ensures that $H\left(\left(\tau_{s}^{+}\right)-\right)=0$. Therefore (5.12) and (5.13) yield

$$
|H(t)-H(s)| \leq K_{T}^{1}(\omega)\left(\sigma\left(\tau_{s}^{+}-s\right)+\sigma\left(t-\tau_{t}^{-}\right)\right) \leq 2 K_{T}^{1}(\omega) \sigma(t-s) .
$$

This proves (1.8) for $t-s \leq 1$, and the full result follows trivially.

If $r \geq 0$ and $v>0$ are fixed, then $H\left(S^{r, v}+t\right)=H^{r, v}(t)$ for $t \leq v$, and therefore

$$
\begin{aligned}
& \underset{t \rightarrow 0+}{\limsup }\left|H \cdot B\left(S^{r, v}+t\right)-H \cdot B\left(S^{r, v}\right)\right|\left(t \log \log \frac{1}{t}\right)^{-1 / 2} \sigma\left(\frac{t^{3}}{2}\right)^{-1} \\
& \quad=\limsup _{t \rightarrow 0+}\left|H^{r, v} \cdot X^{r, v}(t)\right|\left(t \log \log \frac{1}{t}\right)^{-1 / 2} \sigma\left(\frac{t^{3}}{2}\right)^{-1} \\
& \geq 1 \quad \text { a.s. (by Proposition } 5.3(\mathrm{~b})) .
\end{aligned}
$$

(5.1) and (5.2c) complete the proof of (1.9).

ACKNOWLEDGMENT. We thank Terry Lyons for his proof of Lemma 4.4 and a referee for a very careful reading of the paper. The second author thanks the British S.E.R.C. for a visiting fellowship at Cambridge University, during the tenure of which this work was carried out, and NSERC of Canada for financial support.

\section{REFERENCES}

1. M. T. Barlow (1978), Study of a filtration expanded to include an honest time, Z. Wahrsch. Verw. Gebiete 44, 307-323.

2. M. T. Barlow and E. A. Perkins (1985), Sample path properties of stochastic integrals, and stochastic differentiation (preprint).

3. P. Billingsley (1968), Convergence of probability measures, Wiley, New York.

4. B. Davis (1983), On Brownian slow points, Z. Wahrsch. Verw. Gebiete 64, 359-367.

5. B. Davis and E. A. Perkins (1985), Brownian slow points; the critical cases, Ann. Probab. 13, 774-803..

6. C. Dellacherie and P. A. Meyer (1980), Probabilités et potentiel, Hermann, Paris.

7. J. L. Doob (1984), Classical potential theory and its probabilistic counterpart, SpringerVerlag.

8. N. El Karoui and M. Chaleyat-Maurel (1978), Un problème de réflexion et ses applications au temps local et aux équations différentielles stochastiques sur $\mathbf{R}$. Cas continu, Temps Locaux (J. Azéma and M. Yor, eds.), Astérisque 52-53, pp. 117-144.

9. B. Fristedt (1974), Sample functions of stochastic processes with stationary independent increments, Advances in Probability and Related Topics, vol. 3 (P. Ney and S. Port, eds.), Dekker, New York.

10. P. Greenwood and E. A. Perkins (1983), A conditioned limit theorem for random walk and Brownian local time on square root boundaries, Ann. Probab. 11, 227-261.

11. _ (1984), Limit theorems for excursions from a moving boundary, Theory Probab. Appl. 29, 517-528.

12. D. L. Iglehart (1974), Functional central limit theorems for random walks conditioned to stay positive, Ann. Probab. 2, 608-619.

13. K. Itô (1970), Poisson point processes attached to Markov processes, Proc. Sixth Berkeley Sympos., pp. 225-239. 
14. T. Jeulin (1980), Semi-martingales et grossissement d'une filtration, Lecture Notes in Math, vol. 833, Springer-Verlag.

15. J.-P. Kahane (1974), Sur l'irrégularité locale du mouvement Brownien, C. R. Acad. Sci. Paris Ser. A 278, 331-333.

16. H. Kesten (1965), An iterated logarithm law of local time, Duke Math. J. 32, 447-456.

17. F. B. Knight (1981), Essentials of Brownian motion and diffusion, Math. Surveys, vol. 18, Amer. Math. Soc., Providence, R.I.

18. P. A. Meyer (1976), Un cours sur les intégrales stochastiques, Séminaire de Probabilités $\mathrm{X}$, Lecture Notes in Math, vol. 511, Springer-Verlag.

19. E. A. Perkins (1983), On the Hausdorff dimension of the Brownian slow points, Z. Wahrsch. Verw. Gebiete 64, 369-399.

20. A. V. Skorokhod (1961), Stochastic equations for diffusion processes in a bounded region, Theory Probab. Appl. 6, 264-274.

21. K. Uchiyama (1980), Brownian first exit from and sojourn over one sided moving boundary and application, Z. Wahrsch. Verw. Gebiete 54, 75-116.

22. D. Williams (1979), Diffusions, Markov processes, and martingales, Wiley, New York.

23. T. Yamada and S. Watanabe (1971), On the uniqueness of solutions of stochastic differential equations, J. Math. Kyoto Univ. 11, 155-167.

24. M. Yor (1978), Sur la continuité des temps locaux associés a certaines semi-martingales, Temps Locaux (J. Azema and M. Yor, eds.), Astérisque 52-53, pp. 23-35.

25. T. Yamada and Y. Ogura (1981), On the strong comparison theorems for solutions of stochastic differential equations, Z. Wahrsch. Verw. Gebiete 56, 3-19.

26. H. Kunita (1982), Stochastic differential equations and stochastic flows of diffeomorphisms, Cours a l'école d'été de probabilités de Saint-Flour XII.

27. P. Lévy (1939), Sur certains processes stochastiques homogènes, Compositio Math. 7, 283-339.

28. P. A. Meyer, R. T. Smythe and J. B. Walsh (1972), Birth and death of Markov processes, Proc. Sixth Berkeley Sympos., pp. 293-305.

Statistical Laboratory, 16 Mill lane, Cambridge, England CB2 1SB

Department of Mathematics, UNiversity of BRitish Columbia, VANCOUVER, BRITISH COLUmbia, CANADA V6T 1Y4 